(C2020. This manuscript version is made available under the CC-BY-NC-ND 4.0 license http://creativecommons.org/licenses/by-nc-nd/4.0/

\title{
FOAMS OF VEGETABLE OILS CONTAINING LONG-CHAIN TRIGLYCERIDES
}

\author{
Yu Liu and Bernard P. Binks* \\ Department of Chemistry, University of Hull, \\ Hull. HU6 7RX. UK
}

Submitted to: J. Colloid Interface Sci. on 23.6.20 ; revised 11.9.20

Contains ESI

*Corresponding author: $\quad$ b.p.binks@hull.ac.uk

Tel. $01482-465450$

\section{Highlights}

- Stable edible oil foams without added foaming agent

- Air bubbles coated by crystals of long-chain, unsaturated triglycerides

- Packing density of crystals at air bubble surfaces in oil depends on oil type

- Thermo-responsiveness of oil foams

- Chemical composition of high melting triglyceride crystals 


\begin{abstract}
Hypothesis

Can vegetable oils containing long-chain triglycerides be aerated to yield stable oil foams? This is based on the idea that cooling of vegetable oil results in the formation of crystals of certain triglyceride chain lengths and composition dispersed in liquid oil of other chain lengths and composition. Do such oleogels allow the formation of oil foams stabilised by adsorbed crystals?
\end{abstract}

\title{
Experiments
}

Using two vegetable oils, the temperatures for crystal formation are determined. Crystal dispersions were characterised using rheology and optical microscopy. Oleogels were aerated using a double beater and the effects of temperature and aeration time were investigated. The stability and microstructure of the oil foams were studied visually and using microscopy. A stable oil foam was progressively destabilised on heating.

\section{Findings}

Upon cooling/warming vegetable oils, crystals of high melting triglyceride form in a low melting liquid oil - an oleogel. Such oleogels can be whipped to fabricate oil foams stabilised by fat crystals. Optimum foaming yields an over-run of $\sim 40 \%$ for peanut oil and $\sim 110 \%$ for olive oil. Oil foams which do not exhibit drainage, coarsening or coalescence result. We show that high melting triglyceride crystals possess a higher fraction of saturated fatty acids than the original oil. Ultra-stable oil foams can be rendered unstable by heating upon approaching the melting point of the crystals.

Keywords: long-chain triglycerides, crystals, vegetable oil foam, ultra-stable, thermo-responsive 


\section{Introduction}

Comprehensive research on gas-in-oil (i.e. oil foams) is urgently needed given their omnipresence in a number of industries, e.g. food, ${ }^{1}$ petroleum, ${ }^{2,3}$ personal care and pharmaceutical, ${ }^{4}$ but there is a paucity of papers describing the stabilisation mechanisms. The stabilisation of oil foams is quite different from that of aqueous foams. Most oils possess relatively low surface tension $\left(<35 \mathrm{mN} \mathrm{m}^{-1}\right)$ compared to that of water $\left(72 \mathrm{mN} \mathrm{m}^{-1}\right.$ at $\left.25^{\circ} \mathrm{C}\right)$, making the adsorption of most oil-soluble emulsifiers at the oil-air interface energetically unfavourable. ${ }^{4-6}$ Despite this drawback, the stabilisation of oil foams has been achieved in a number of ways. Firstly, fluorocarbon-based surfactants have a lower surface energy than hydrocarbon-based ones, being both hydrophobic and oleophobic, thereby making them potential candidates to stabilize both aqueous and non-aqueous foams. ${ }^{7}$ Bergeron et al. ${ }^{8}$ found that the adsorption of fluorocarbon surfactant molecules at the dodecane-air interface led to a reduction of the surface tension. The stability of bulk foams and foam films was ensured by a repulsive disjoining pressure arising from the overlap of surfactant layers. The role of the solubility phase boundary of a solute in oil on foaming was first elucidated by Ross and Nishioka. ${ }^{9}$ They found a significant increase in foam stability when approaching the solubility limit of the stabilising agent in oil, i.e. as it became insoluble. This was further verified by Friberg et al. ${ }^{10}$ on investigating the dispersion and foaming behavior of triethanolammonium oleate in $p$-xylene. Later, by studying the foaming properties of a series of low molar mass and polymeric surfactants in poly(decene) oil, Binks et al. ${ }^{11}$ demonstrated enhanced foamability near the phase separation boundary of the solute in oil where the affinity of the former for the latter was low. However, it was only recently that the mechanism behind the foaming of complex lubricant base oils was further unveiled, due to solutocapillary flow originating from differential evaporation of the different lubricant components. ${ }^{12}$ Foams from mono- and diglycerol fatty acid esters in long-chain alkanes and vegetable oils were systematically studied by Shrestha and coworkers. ${ }^{13-16}$ Foamability and foam stability were determined by the surfactant self-assembled aggregates formed within the two-phase region, being affected by the oil nature, surfactant concentration, surfactant architecture and temperature. Surfactant solids $(\alpha$ or $\beta)$ were shown to be better foam stabilizers than the lamellar liquid crystalline phase $\left(L_{\alpha}\right)$.

Oil foams can also be stabilized solely by colloidal particles. Of relevance, early work by Fox and Zisman ${ }^{17,18}$ discussed the wetting behaviour of a variety of oils on smooth substrates of low surface energy in air, e.g. poly(tetrafluoroethylene) (PTFE) and modified 
tetrafluoroethylene polymers. Subsequently, Murakami and co-workers ${ }^{19,20}$ succeeded in preparing both oil foams and dry oils with oligotetrafluoroethylene (OTFE) particles. Systematic investigations carried out by Binks et al. ${ }^{21-25}$ optimised the conditions for the stabilization of air-oil interfaces by a number of solid particle types. The investigated particles were fluoroethylene, ${ }^{21,22}$ fluorinated fumed silica, ${ }^{23}$ fluorinated clay $^{24}$ and other fluorinated particles. ${ }^{25}$ The type of material formed (viz. oil foam, dry oil, oil liquid marble) was influenced by the oil surface tension, the particle surface energy and the level of mechanical energy input.

Recently, oil foams find applications in the food industry because of their potential in reducing saturated fat intake, providing more desirable mouthfeel and reducing delivery costs. ${ }^{1,26}$ The approaches for preparing ultra-stable edible oil foams are developed on the basis of oleogels, during which a homogeneous solution of edible emulsifier in vegetable oil is first submitted to a specific tempering procedure to yield an oleogel, followed by mechanical energy input to generate an air-in-oil foam stabilized by pre-formed crystals. ${ }^{5,26}$ The potential stabilizing agents can be crystals of mono- and/or diglycerides, ${ }^{27-31}$ fatty alcohols ${ }^{32}$ fatty acids, ${ }^{33}$ mixtures of phytosterols and monoglycerides ${ }^{34}$ and high-melting triglycerides (TAGs). ${ }^{35-39}$ Brun et al. ${ }^{27}$ reported the fabrication of ultra-stable oil foams in the system of mono- and diglycerides in rapeseed oil. The resulting foams were stable against coalescence and Ostwald ripening as a result of a rigid layer of crystals surrounding the bubbles. Moreover, the crystal network structure was firm enough to arrest gravity-driven creaming. In another study, Gunes et al. ${ }^{28}$ pointed out that the application of whipping was necessary for the creation of a compact layer of crystals at the bubble surface, thereby providing Pickering-type interfacial stabilization. In addition, foamability was shown to be closely related to the gel rheology prior to whipping. The first study on the stabilization of vegetable oil foams by high-melting fat crystals was reported by Mishima et al. ${ }^{35}$ Small $\beta$-fat crystals were proven to be a pre-requisite for preparing well-whipped salad oil. More importantly, the arrangement of TAG crystals near the air-oil interface was determined by synchrotron radiation microbeam X-ray diffraction (SR- $\mu-\mathrm{XRD})$. Later, Binks and Marinopoulos ${ }^{36}$ reported on the foaming potential of neat coconut oil and other vegetable oils composed of medium chain fatty acids, in the absence of any additive. Oil foams are not necessarily the final products, however. Most recently, oil foams formed from anhydrous milk fat were used as intermediates to prepare air-in-oil-in-water emulsions, which paved the way for developing novel oil foam-based colloidal systems with new functionalities. ${ }^{37}$ All the above mentioned cases involving the preparation of edible oil foams were dependent on pre-formed crystals. 
Up to now, investigations on oil foams stabilized by high-melting fat crystals are still limited, and the stabilization mechanisms are far from well understood. In the present study, we explore the possibility of foaming neat vegetable oils containing long-chain, unsaturated fatty acids, i.e. refined peanut oil and extra virgin olive oil, in the absence of any added foaming agent. The size, morphology, polymorph type and concentration of crystals play a dominant role in foam formation and stabilisation, ${ }^{5,26,38}$ so we first apply different temperature-time dependent tempering protocols to induce the formation of high-melting fat crystals within a continuous liquid oil phase. Different methods including visual observation, differential scanning calorimetry, solid fat content, rheology and microscopy are utilised to give insight into the characteristics of crystal dispersions or oil gels before whipping. Subsequently, the foaming efficiency and stability are evaluated as a function of aeration temperature, and further correlated to the physicochemical properties of samples before whipping. High-melting fat crystals contributing to foaming are extracted and analysed by GC/MS. Finally, we investigate the influence of temperature on an initially ultra-stable foam as it approaches the melting point of triglyceride crystals.

\section{Experimental}

\subsection{Materials}

Refined peanut oil, RPO, was from Eulip, Italy. It is a clear, light yellow liquid at ambient temperature $\left(23 \pm 3{ }^{\circ} \mathrm{C}\right)$. Extra virgin olive oil, EVOO, was a gift from Cargill NV, Belgium (SPLI-72120). It is a dark yellow liquid at room temperature. Their fatty acid composition in the form of methyl esters (FAMEs) was measured by gas chromatography-mass spectrometry (GC/MS). RPO is abundant in long-chain unsaturated fatty acids (UFAs), i.e. oleic (C18:1, $49.7 \%, \mathrm{O})$ and linoleic $(\mathrm{C} 18: 2,22.8 \%, \mathrm{~L})$. It also contains saturated fatty acids (SFAs) being palmitic $(\mathrm{C} 16: 0,12.0 \%, \mathrm{P})$, behenic $(\mathrm{C} 22: 0,3.5 \%, \mathrm{Be})$, stearic $(\mathrm{C} 18: 0,3.0 \%$, S), lignoceric $(\mathrm{C} 24: 0,1.9 \%, \mathrm{Lg})$ and arachidic $(\mathrm{C} 20: 0,1.6 \%, \mathrm{~A})$. In the case of EVOO, long-chain UFAs dominate as well, being oleic (79.9\%) and palmitoleic (C16:1, 0.9\%, Po). It contains $\sim 17.0 \%$ SFAs in total. The fatty acid composition of both oils was comparable to that reported previously. ${ }^{40,41}$ Potassium hydroxide $(\geq 85 \%)$ was obtained from Fisher Chemicals. Methanol $(\geq 99.8 \%)$ was purchased from Honeywell and $n$-hexane $(\geq 97 \%)$ was from VWR Chemicals. All materials were used as received unless stated otherwise.

\subsection{Methods}




\subsubsection{Cooling and warming oils}

The oil sample in a glass vial $(75 \mathrm{~mm} \times 25 \mathrm{~mm}$, Fisher $)$ with a screw cap was placed into the well of a thermostat (VWR International) equipped with a MX07R-20 temperature controller. The thermostatting liquid was a homogeneous mixture of ethylene glycol and deionized water. All temperatures were measured by an Omega thermocouple $\left( \pm 0.1{ }^{\circ} \mathrm{C}\right)$. Oil samples were pre-heated at $60{ }^{\circ} \mathrm{C}$ for $\sim 30 \mathrm{~min}$ in the thermostat to erase any history. Subsequently, they were submitted to different thermal treatment: (i) gradual cooling from $60{ }^{\circ} \mathrm{C}$ to the temperature of interest; (ii) rapid cooling to $-20{ }^{\circ} \mathrm{C}$ and maintaining at this temperature overnight $(\sim 12 \mathrm{~h})$, followed by slow heating to the target temperature.

\subsubsection{Differential scanning calorimetry (DSC)}

The crystallisation and melting profiles of the oils were investigated using a Differential Scanning Calorimeter (Mettler 822e) with $\mathrm{STAR}^{\mathrm{e}}$ software. Nitrogen was used as both purge and drying gas. The instrument was calibrated with indium. The oils $(5-10 \mathrm{mg})$ were weighed using an analytical balance (Mettler Toledo), then hermetically sealed into $40 \mu \mathrm{L}$ aluminium pans (ME-26763) at room temperature. Another empty, hermetically sealed aluminium pan of the same type was used as the reference. The applied cooling and warming program was: (i) holding isothermally at $20{ }^{\circ} \mathrm{C}$ for $10 \mathrm{~min}$; (ii) cooling from $20{ }^{\circ} \mathrm{C}$ to $-20{ }^{\circ} \mathrm{C}$ at $0.5{ }^{\circ} \mathrm{C} \mathrm{min}^{-1}$; (iii) holding isothermally at $-20{ }^{\circ} \mathrm{C}$ for $10 \mathrm{~min}$; (iv) warming from $-20{ }^{\circ} \mathrm{C}$ to $20{ }^{\circ} \mathrm{C}$ at $0.5{ }^{\circ} \mathrm{C}$ $\min ^{-1}$. All tests were performed in triplicate.

\subsubsection{Solid fat content (SFC)}

The measurement of the solid fat content (SFC) of RPO at different temperatures was performed using a Bruker NMR minispec mqone SFC analyser, the principle of which was explained elsewhere. ${ }^{42}$ First, tubes containing liquid oils were placed on a heating block at 80 ${ }^{\circ} \mathrm{C}$ for $10 \mathrm{~min}$, and then transferred to a $0{ }^{\circ} \mathrm{C}$ thermostat and maintained for $1 \mathrm{~h}$. After those pre-conditions, the samples were kept at each temperature for at least $30 \mathrm{~min}$ prior to measurement. Groundnut oil from AAK (Hull), also known as peanut oil, was measured for comparison.

\subsubsection{Optical microscopy}

A Leica DME optical microscope mounted with a Leica MC190 HD camera was used to capture optical micrographs of crystal dispersions in oil and air-in-oil foams. A polarizer (13596080) and an analyzer were used to cross-polarize the transmitted light. Images were 
acquired by Leica Application Suite 4.12.0. A small amount of sample was transferred carefully by spatula onto the middle of a glass slide $(76 \mathrm{~mm} \times 26 \mathrm{~mm})$ possessing a single cavity $(15 \mathrm{~mm})$, and then gently covered with a thin coverslip $(24 \mathrm{~mm} \times 24 \mathrm{~mm})$. The temperature of the glass slide was controlled by a hot stage (Linkam PE120), which was connected to an ECP water circulator and controlled by a Linkam T95PE controller. A moderate flow rate of dry compressed air was applied over the coverslip during imaging to avoid condensation. Both polarized and non-polarized microscope images were taken for the same sample under the same condition at fixed time intervals during both whipping (foams) and storage. All glassware and spatulas were pre-cooled at the whipping or storage temperature before use. The micrographs were analyzed with Image J $1.47 \mathrm{~V}$ software which was calibrated with a Pyser-sgi limited graticule. The number average bubble diameter $D[1,0]$ of the aerated samples was calculated from at least 100 representative bubbles of different samples:

$$
\mathrm{D}[1,0]=\frac{\sum_{i=1}^{n} d_{i}}{n}
$$

where $d_{\mathrm{i}}$ is the bubble diameter and $n$ is the number of bubbles.

\subsubsection{Cryo-scanning electron microscopy (Cryo-SEM)}

A drop of fresh oil foam was mounted onto a channelled aluminium stub with a spatula. The sample was immersed into liquid nitrogen for $2 \mathrm{~min}$ before being transferred to a cryo-preparation chamber (PP3010T, Quorum Technologies Ltd). The temperature of the preparation stage was $-140{ }^{\circ} \mathrm{C}$. A scalpel was used to fracture the frozen sample exposing a freshly cut surface, which was then coated with platinum ( $\sim 2 \mathrm{~nm}$ film thickness). The sample was transferred to the cold stage at $-140{ }^{\circ} \mathrm{C}$ in a Carl Zeiss Evo-60 SEM instrument and imaged. Imaging was performed with a LaB6 emitter, 40 micro-amp beam current, $15 \mathrm{kV}$ accelerating voltage and a 35 pico-amp probe current using the secondary electron detector.

\subsubsection{Rheology}

A Bohlin rheometer (CVO120 High Resolution) equipped with software (Bohlin R6.51.0.3) was used for rheological measurements. To prevent wall slip, serrated parallel plates of 
diameter $40 \mathrm{~mm}$ were used. The gap between the upper and lower plates was $1 \mathrm{~mm}$. The temperature of the lower plate was precisely controlled by a Peltier system $\left( \pm 0.1{ }^{\circ} \mathrm{C}\right)$. To determine the evolution of the elastic modulus $\left(G^{\prime}\right)$ and viscous modulus $\left(G^{\prime \prime}\right)$ with temperature under small deformation oscillation mode, a temperature sweep was performed from $60{ }^{\circ} \mathrm{C}$ to $-20{ }^{\circ} \mathrm{C}$ or lower, then maintained isothermally at this temperature for $10 \mathrm{~min}$, followed by warming gradually to $60{ }^{\circ} \mathrm{C}$ with $f=0.1 \mathrm{~Hz}$. The cooling and heating rate was 1 ${ }^{\circ} \mathrm{C} \min ^{-1}$, and the oscillation strain $\gamma$ was set as $0.05 \%$ within the linear viscoelastic region (LVR) of oil gels (Figure S1). ${ }^{43}$ To better understand the crystal network development upon cooling and warming, four critical temperatures were identified from the temperature ramp curves as: $T_{c c}$ - crossover temperature $\left(G^{\prime}=G^{\prime \prime}\right)$ of crystallisation during cooling; $T_{o c}$ - onset temperature of crystallisation during cooling; $T_{c m}$ - crossover temperature $\left(G^{\prime}=G^{\prime \prime}\right)$ of crystal melting during warming; $T_{e m}$ - end temperature of crystal melting during warming. ${ }^{32}$ The relaxation behaviour of oil gels was investigated by a frequency sweep $(0.01 \mathrm{~Hz}-10 \mathrm{~Hz})$ with $\gamma=0.05 \%$. Amplitude sweeps were carried out at representative temperatures at a fixed frequency of $0.1 \mathrm{~Hz}$. The measuring geometry was cleaned with ethanol before use.

\subsubsection{Preparation and characterisation of oil foams}

A glass beaker containing $100 \mathrm{~mL}$ of RPO or EVOO was initially maintained at $60{ }^{\circ} \mathrm{C}$ in the well of the thermostat to erase history. In the case of the cooling protocol, the oil sample was cooled slowly at a rate of $0.3{ }^{\circ} \mathrm{C} \mathrm{min}^{-1}$ until reaching the required temperature. In contrast, the sample submitted to the warming procedure was heated at $0.4{ }^{\circ} \mathrm{C} \min ^{-1}$ to temperatures of interest after pre-conditioning at $-20{ }^{\circ} \mathrm{C}$ overnight. This was followed by whipping with a hand-held Vonshef double beater electric whisk at level $1(750 \pm 75 \mathrm{rpm})$. The whole whipping procedure lasted for $45 \mathrm{~min}$ in total containing $5 \mathrm{~min}$ whipping followed by $5 \mathrm{~min}$ rest interval, i.e. actual whipping time was 25 min. $^{33,36}$ Both digital photos and microscope images were taken at rest intervals. Immediately after whipping the homogeneous sample was quickly transferred into two small glass beakers pre-cooled to the aeration temperature. One was stored at the aeration temperature to monitor foam stability (i.e. volume of foam, volume of drained oil and foam bubble size versus storage time), whilst the other was used for calculating the over-run of the whipped oil. The initial volume of the aerated sample $\left(V_{\text {oil }+ \text { air }}\right.$ ) equals the volume of oil plus entrapped air bubbles, and after warming to room temperature $\left(23 \pm 3{ }^{\circ} \mathrm{C}\right)$ to fully release air bubbles the sample was returned to its initial 
whipping temperature at which the volume of oil $\left(V_{\text {oil }}\right)$ before whipping was determined. The over-run is calculated as:

$$
\text { over }-\operatorname{run}(\%)=\frac{V_{\text {oiltair }}-V_{\text {oil }}}{V_{\text {oil }}} \times 100 \%
$$

For comparison, EVOO was passed twice through a basic alumina column to remove potential polar impurities. Subsequently, the columned EVOO was subjected to the same warming procedure followed by the same whipping protocol mentioned above. All glassware was washed with liquid detergent, rinsed with ethanol and Milli-Q water, and fully dried in an oven before use. All glass beakers were sealed with aluminium foil during both whipping and storage to avoid condensation.

\subsubsection{Separation of crystals by filtration}

The oil was maintained at $-20{ }^{\circ} \mathrm{C}$ overnight, followed by warming gradually to $8 \pm 1{ }^{\circ} \mathrm{C}$ to yield a crystal dispersion. Subsequently, it was filtered through a filter disc (Sartorius 393, diameter: $70 \mathrm{~mm}$, pore size: $1 \mu \mathrm{m}$ ) fitted in a Büchner funnel with a vacuum flask, which were connected to a portable vacuum pump (Diaphragm MZ 2C, Germany). ${ }^{44,45}$ The whole filtration process was carried out in a cool room of $7 \pm 1{ }^{\circ} \mathrm{C}$. The initial solvent-free vacuum filtration lasted for $\sim 30 \mathrm{~min}$, and the filtrate was washed gently with $n$-hexane to remove any entrained liquid oil and left to dry overnight.

2.2.9 Synthesis of FAMEs and analysis by gas chromatography-mass spectrometry (GC/MS) FAMEs were obtained through the catalytic trans-esterification method as described by the EU official method. ${ }^{46}$ The FAME samples were submitted to a 1:100 dilution with $n$-hexane before GC/MS analysis. The FAME composition was separated in a Restek Rxi-5 MS capillary column $(30 \mathrm{~m}($ length $) \times 0.25 \mathrm{~mm}($ internal diameter $) \times 0.25$ um (film thickness $))$ in an Agilent 6890+ GC coupled to an Agilent 5973N MSD quadrupole MS. The oven temperature was held initially at $70{ }^{\circ} \mathrm{C}$ for $1 \mathrm{~min}$ after injection, and adjusted to $300{ }^{\circ} \mathrm{C}$ at 15 ${ }^{\circ} \mathrm{C} \mathrm{min}^{-1}$ and held for $30 \mathrm{~min}$. The sample was injected at the split injection mode with a split ratio of 25:1, and the carrying gas was helium at a rate of $1 \mathrm{~mL} \mathrm{~min}{ }^{-1}$. The injector temperature was $260{ }^{\circ} \mathrm{C}$ and the injection volume was $1 \mu \mathrm{L}$. MS detector conditions were positive mode electron ionisation at $70 \mathrm{eV}$, ion source temperature at $210{ }^{\circ} \mathrm{C}$, quad temperature at $150{ }^{\circ} \mathrm{C}$. The MS spectrum was compared with data from the NIST library.

\subsubsection{Statistical analysis}


Statistical analysis was performed using IBM SPSS Statistics (version 25). Data were expressed as average values \pm standard deviations of the mean $(n \geq 3)$. Comparisons were made using t-test and $p$-values $<0.05$ were considered significantly different.

\section{Results and Discussion}

We firstly examine the crystallisation and melting behaviour of the two oils using different methods. Secondly, their foamability and foam stability are studied at representative temperatures during both cooling and warming. Thirdly, the thermo-responsiveness of an otherwise stable oil foam is demonstrated. Finally, the fatty acid composition of extracted high-melting fat crystals responsible for foaming is determined by GC/MS, and compared to that of the initial liquid oil.

\subsection{Crystallisation and melting behaviour of vegetable oils}

\subsubsection{Appearance}

The effect of temperature on the crystallisation and melting of the oils was preliminarily investigated by visual observations. The samples were either cooled gradually from $60{ }^{\circ} \mathrm{C}$ at $0.3{ }^{\circ} \mathrm{C} \mathrm{min}^{-1}$ or warmed at $0.4{ }^{\circ} \mathrm{C} \mathrm{min}-1$ from $-20{ }^{\circ} \mathrm{C}$. Photos of RPO at representative temperatures upon cooling and warming are shown in Figure 1(a) and Figure $\mathrm{S} 2(\mathrm{a})$, respectively. On cooling, it is a clear, yellowish liquid above $5{ }^{\circ} \mathrm{C}$. The first evidence of crystallisation appears at $4{ }^{\circ} \mathrm{C}\left(T_{\text {crystal,onset }}\right)$ where the sample turns slightly cloudy. With further supercooling ( $\Delta T=T-T_{\text {crystal,onset }}$, where $T$ is the target temperature $)^{39}$, it becomes more turbid and viscous. On reaching the minimum gelling temperature $\left(T_{\mathrm{g}}\right)^{47}$ of $\sim-5{ }^{\circ} \mathrm{C}$, the sample does not flow when the vessel is inverted. There is no further change in sample appearance when cooling down to $-20{ }^{\circ} \mathrm{C}$. Upon warming, the sample remains gelled up to $3{ }^{\circ} \mathrm{C}$, although it gradually softens. A noticeable change occurs at $\sim 4{ }^{\circ} \mathrm{C}$ where it becomes a flowing viscous dispersion. As the temperature increases further, the sample becomes less turbid, and finally transits back to a clear liquid at $13{ }^{\circ} \mathrm{C}$ and higher.

The appearance evolution of EVOO with temperature is illustrated in Figure 1(b) and Figure S2(b). As expected, the first sign of solidification occurs at a lower temperature compared to RPO, i.e. $\sim-9{ }^{\circ} \mathrm{C}$, due to a higher proportion of UFAs in the former. It then develops into an oil gel at $-12{ }^{\circ} \mathrm{C}$, a few ${ }^{\circ} \mathrm{C}$ lower than the crystallisation temperature $T_{\text {crystal,onset }}$. When heating, the transition from a firm gel to a flowing dispersion occurs around $8{ }^{\circ} \mathrm{C}$, followed by the appearance of a clear, dark yellow liquid at $11^{\circ} \mathrm{C}$. Hysteresis exists 
between cooling and warming of both oils, as reported earlier. ${ }^{36}$ This is due to the fact that triglyceride crystal formation triggered by supercooling involves an initial stage of nucleation followed by crystal growth which is absent for crystal melting. ${ }^{48,49}$ Additionally, the transitions between different crystal polymorphs induced by different tempering protocols may account for this difference. ${ }^{48,49}$ The driving force for fat crystallization can be characterised by the difference between the crystallization temperature and the global melting temperature, i.e. the highest temperature at which solid phases can survive upon warming. ${ }^{49}$ It appears that the driving force for EVOO crystallisation $\left(\sim 19^{\circ} \mathrm{C}\right)$ is significantly larger than that for RPO crystallisation $\left(\sim 8^{\circ} \mathrm{C}\right)$.

\subsubsection{Thermal properties and SFC}

Thermal analysis was carried out using DSC as shown in Figure 2(a) for RPO and Figure 2(b) for EVOO. In the case of RPO, the first exothermic event occurs at $2.6{ }^{\circ} \mathrm{C}$ during cooling, implying the onset of crystallisation. One distinct exothermic peak is observed at $-0.2{ }^{\circ} \mathrm{C}$ which is mainly attributed to the crystallization of high-melting, di-saturated triglyceride fractions, ${ }^{50,51}$ e.g. PPL, PPO, PSL, PSO, PLBe. ${ }^{52}$ Three distinguishable endothermic peaks can be detected from the melting curve. The prominent one occurs at 10.5 ${ }^{\circ} \mathrm{C}$, whereas the two minor ones are at $-16.9{ }^{\circ} \mathrm{C}$ and $-2.6{ }^{\circ} \mathrm{C}$, respectively. The crystallisation and melting profiles here are in close agreement with those determined by Chen Man and Tan. ${ }^{53,54}$ Similarly, the cooling thermogram of EVOO displays one distinct exothermic peak but at a lower temperature of $-14.3{ }^{\circ} \mathrm{C}$, and the first crystallisation event starts at -11.5 ${ }^{\circ} \mathrm{C} .{ }^{50,51,53,54}$ Upon heating, two well-defined endothermic peaks are identified at $6.6{ }^{\circ} \mathrm{C}$ and $-6.0{ }^{\circ} \mathrm{C}$. A less evident endothermic peak is also observed at $2.6{ }^{\circ} \mathrm{C}$. The thermal properties of RPO and EVOO are summarised in Table S1.

In order to quantify the mass fraction of fat crystals at different temperatures for one of the oils, pulsed-NMR was applied. Figure 2(c) shows that the SFC values of RPO and groundnut oil increase with a decrease in temperature, especially at temperatures lower than $10{ }^{\circ} \mathrm{C}$. This is due to the fact that a higher degree of supercooling tends to accelerate the kinetics of trigyceride nucleation and growth. ${ }^{48,49}$ The minimum SFC to form an oil gel is $\sim 4.5 \%$, similar to the values $(\leq 5 \%)$ of other oleogel systems..$^{33,47,55}$

\subsubsection{Crystal morphology and rheological properties}

Figure 3(a) presents polarised micrographs of RPO obtained at different temperatures cooled slowly from $60{ }^{\circ} \mathrm{C}$. RPO crystals are revealed as needle-like in geometry, as observed 
previously with groundnut oil, ${ }^{56}$ of size smaller than $10 \mu \mathrm{m}$. With decreasing temperature more crystals tend to form and disperse within the isotropic liquid oil due to a higher degree of supercooling. Upon reaching temperatures below $T_{\mathrm{g}}$ of $-5{ }^{\circ} \mathrm{C}$, individual fat crystals interact with each other mainly through van der Waals forces, producing a three-dimensional crystal network or oil gel. EVOO crystals are different to those of RPO, as seen in Figure 3(b). No crystal forms at $-6{ }^{\circ} \mathrm{C}$ (not shown), but further cooling leads to polydisperse spherical crystals of size between 5 and $40 \mu \mathrm{m}$ with characteristic Maltese crosses, reminiscent of anhydrous milk fat crystals reported by Goibier et al. ${ }^{37}$

The rheological properties of neat oils were investigated by cooling from $60{ }^{\circ} \mathrm{C}$ to -20 ${ }^{\circ} \mathrm{C}$ or lower at $1{ }^{\circ} \mathrm{C} \mathrm{min}^{-1}$, maintaining isothermally at low temperature for $10 \mathrm{~min}$ before warming to $60{ }^{\circ} \mathrm{C}$ at $1{ }^{\circ} \mathrm{C} \mathrm{min}^{-1}$ in a temperature-controlled rheometer using parallel plates. The viscoelasticity $\left(G^{\prime}\right.$ and $\left.G^{\prime \prime}\right)$ of RPO is recorded as a function of temperature in Figure 4(a). During cooling, initially both $G^{\prime}$ and $G^{\prime \prime}$ are low $(<0.1 \mathrm{~Pa})$ with $G^{\prime}<G^{\prime \prime}$ corresponding to a weak liquid. A sudden increase in both parameters at $\sim 2{ }^{\circ} \mathrm{C}$ denotes the onset of crystallisation, followed by a monotonic increase with decreasing temperature during which the sample transforms gradually from liquid-like $\left(G^{\prime}<G^{\prime \prime}\right)$ to solid-like $\left(G^{\prime}>G^{\prime \prime}\right)$. The extracted crossover temperature of $G^{\prime} / G^{\prime \prime}\left(T_{\mathrm{cc}}\right)$ is comparable to $T_{\mathrm{g}}$ obtained from visual observations. Finally, for temperatures $<-8{ }^{\circ} \mathrm{C}, G$ increases slowly until it reaches around $5 \times 10^{5} \mathrm{~Pa}$. In this region, the value of $G$ " is approximately one order of magnitude smaller than $G^{\prime}$, and almost independent of temperature. This viscoelastic development is similar to that reported by Ojijo et al. ${ }^{57}$ for monoglyceride/olive oil mixtures. Upon heating from -20 ${ }^{\circ} \mathrm{C}, G^{\prime}$ and $G$ " firstly exhibit a progressive decrease, followed by an abrupt decrease until eventually remaining constant in the range of $0.1 \mathrm{~Pa}$. The sample deteriorates from an elastic solid $\left(G^{\prime}>G^{\prime \prime}\right)$ into a viscous liquid with $\left(G^{\prime}<G^{\prime \prime}\right)$ during heating, arising from the gradual melting of triglyceride crystals. The variation of $G^{\prime}$ and $G^{\prime \prime}$ with temperature for EVOO is shown in Figure 4(b). EVOO is a weak liquid initially with $G^{\prime}$ and $G^{\prime \prime}<0.1 \mathrm{~Pa}$. Crystallisation starts at $\sim-9{ }^{\circ} \mathrm{C}$ as evidenced by a sudden increase in both parameters. Subsequently they increase significantly down to the final temperature of $-28{ }^{\circ} \mathrm{C}$, and are still developing during the isothermal period. When submitted to heating, $G$ ' remains almost constant at temperatures $<-11^{\circ} \mathrm{C}$ with $G^{\prime}>G^{\prime \prime}$ possibly arising from the absence of melting events (see Figure 2(b)), which is different from the melting behaviour of RPO. Further heating leads to an abrupt decrease in $G^{\prime}$ and $G^{\prime \prime}$, and finally a liquid-like state where both values are $<0.1 \mathrm{~Pa}$. Based on the above analysis, we conclude that the crystallisation and melting behaviour determined by different methods are in agreement. 


\subsection{Preparation and characterisation of vegetable oil foams}

Oil samples of $100 \mathrm{~mL}$ were either cooled from $60{ }^{\circ} \mathrm{C}$ or warmed from $-20{ }^{\circ} \mathrm{C}$ to temperatures of interest. Subsequent whipping was applied with a double beater electric whisk for $45 \mathrm{~min}$ in total, i.e. 5 min whipping followed by $5 \mathrm{~min}$ rest interval. We first evaluated the foaming ability as a function of whipping time. Then, the effect of whipping temperature on both foamability and foam stability was studied.

\subsubsection{Effect of whipping time}

When subjected to cooling, the aeration of RPO was first carried out at four representative temperatures $\left(6{ }^{\circ} \mathrm{C}, 3{ }^{\circ} \mathrm{C},-5{ }^{\circ} \mathrm{C}\right.$ and $\left.-21.5{ }^{\circ} \mathrm{C}\right)$ corresponding to different stages of crystallisation (liquid, fluid dispersion, soft gel and firm gel respectively). Figure 5(a) and Figure S3(a) present the appearance evolution of aerated RPO at $3{ }^{\circ} \mathrm{C}$ and at the other temperatures, respectively. Clear oil at $6{ }^{\circ} \mathrm{C}$ is unwhippable, with no change in volume upon whipping. For the fluid crystal dispersion at $3{ }^{\circ} \mathrm{C}$ and the soft gel at $-5{ }^{\circ} \mathrm{C}$, a milky white appearance becomes more pronounced as whipping proceeds due to an increasing extent of air incorporation. At $-21.5{ }^{\circ} \mathrm{C}$ however, whipping initially involves breaking the firm gel into a viscous paste, followed by introducing air into the viscoelastic matrix. In the case of EVOO, the appearance with whipping time at selected temperatures during cooling can be seen in Figure 5(b) and Figure S3(b). Between $-4{ }^{\circ} \mathrm{C}$ and $-8{ }^{\circ} \mathrm{C}$, the starting samples are clear, dark yellow liquids. Upon cooling gradually from $-10{ }^{\circ} \mathrm{C}$ to $-14{ }^{\circ} \mathrm{C}$, they develop from a slightly turbid dispersion to a soft gel and then a firm gel. Upon whipping, no change in appearance occurs at $-4{ }^{\circ} \mathrm{C}$. For lower temperatures, the samples are initially yellow and become increasingly white as more air is incorporated. To quantitatively describe the extent of air incorporation during whipping, Figure 5(c) and Figure 5(d) plot the over-run as a function of whipping time for RPO and EVOO, respectively. For the former system, the over-run increases at first and then levels off at $-21.5^{\circ} \mathrm{C}$ and $-5{ }^{\circ} \mathrm{C}$, while for $3{ }^{\circ} \mathrm{C}$ the value increases continuously up to $45 \mathrm{~min}$, similar to the aeration behaviour of coconut oil at 23 ${ }^{\circ} \mathrm{C} .{ }^{36}$ The over-run is zero at $6{ }^{\circ} \mathrm{C}$ where no fat crystals are present, in agreement with the non-foaming behaviour of coconut oil ${ }^{36}$ and anhydrous milk fat ${ }^{37}$ above their crystallisation temperatures. Thus, molecules of triglyceride in these oils are not surface-active at the oil-air surface. The variation of the over-run of EVOO with whipping time is also temperature dependent. At $-8{ }^{\circ} \mathrm{C}$ the over-run increases progressively with whipping time. At all other 
temperatures except $-4{ }^{\circ} \mathrm{C}$, the over-run exhibits a significant increase up to $35 \mathrm{~min}$, followed by no further change.

For a cold sample of RPO subjected to warming, their appearance and over-run values during whipping are given in Figure S4. Similar to the whipping of firm gel at $-21.5{ }^{\circ} \mathrm{C}$ during cooling, the energy input from the double beater is mainly involved in softening the gel prior to enabling effective air incorporation at $-20{ }^{\circ} \mathrm{C}$. At the other temperatures, foaming is achieved once whipping is started. The over-run values of whipped RPO at $3{ }^{\circ} \mathrm{C}$ show a gradual increase with aeration time, whereas for the fluid dispersion at $6{ }^{\circ} \mathrm{C}$ it increases to a plateau followed by a slight decrease as a result of inefficient network stabilisation in the continuous oil phase against drainage and coalescence during whipping. ${ }^{28}$ At the other two temperatures, the over-run increases up to $15 \mathrm{~min}$ and then remains almost constant up to 45 min. The maximum over-run is $\sim 38 \%$ at $-20{ }^{\circ} \mathrm{C}$. For EVOO during heating (see Figure S5), at $-12{ }^{\circ} \mathrm{C}$ and $-6{ }^{\circ} \mathrm{C}$ the first $15 \mathrm{~min}$ whipping serves to soften the firm gel, but subsequent whipping is responsible for effective air incorporation. The soft oil gels at $0{ }^{\circ} \mathrm{C}$ and $6{ }^{\circ} \mathrm{C}$ can be whipped easily, and the over-run values increase up to $15 \mathrm{~min}$ then remain constant. No air incorporation occurs at $12{ }^{\circ} \mathrm{C}$ as expected. The maximum over-run is $\sim 110 \%$ at $-12{ }^{\circ} \mathrm{C}$.

\subsubsection{Effect of whipping temperature}

The over-run of aerated RPO immediately after 45 min whipping is plotted as a function of aeration temperature in Figure 6(a) for samples cooled and warmed. The inset in Figure $6(\mathrm{a})$ is a photograph of RPO foam produced at $-15^{\circ} \mathrm{C}$ upon cooling. Not surprisingly, no foam is obtained above the crystallisation temperature during cooling (filled points) since triglyceride molecules are not surface-active at the air-oil interface. ${ }^{35-37}$ A marked maximum in foamability occurs at $\sim 3{ }^{\circ} \mathrm{C}$ from a fluid crystal dispersion where the degree of supercooling $\Delta T=-1{ }^{\circ} \mathrm{C} .{ }^{39}$ This is line with the findings of Heymans et al. ${ }^{29}$ where the best foaming was achieved from partially crystallised monoglyceride in sunflower oil, but contradicts the conclusions of Gunes et al. ${ }^{28}$ where weak gels were unable to provide effective continuous oil phase stabilisation against drainage and coalescence during whipping for monoglyceride/high oleic sunflower oil mixtures. Some plausible explanations exist for this discrepancy. Firstly, a less viscous crystal dispersion enables more effective air incorporation during whipping. ${ }^{58}$ Secondly, the introduction of new air-oil interfaces upon whipping can provide locations where nucleation and crystal growth of triglyceride molecules can occur, thus protecting the incorporated air bubbles. ${ }^{59,60}$ Last but not least, our observations can be rationalised by an abrupt increase in air-oil surface tension near the onset 
of crystallisation as described by Mishra et $a l .{ }^{39}$ arising from the adsorption of solid triglyceride. When the sample was cooled below $3{ }^{\circ} \mathrm{C}$, we notice a dramatic decrease in over-run until near $T_{\mathrm{g}}\left(-5^{\circ} \mathrm{C}, p<0.05\right)$. This is because a significant increase in the viscosity of the continuous phase hinders air incorporation. ${ }^{33,58}$ Further cooling down to $-21.5{ }^{\circ} \mathrm{C}$ results in a noticeable increase in over-run from $\sim 10 \%$ to $\sim 37 \%(p<0.05)$. The effect of gel rheology on the foaming of monoglycerides in vegetable oils was studied by Gunes et $a l^{28}$ and Heymans et al. ${ }^{29}$ The former found that the most suitable range of $G^{\prime}$ for foaming was $10^{5}-10^{7} \mathrm{~Pa}$, whereas the latter found that a gradual increase in $G^{\prime}$ from $10^{3} \mathrm{~Pa}$ to $10^{5} \mathrm{~Pa}$ resulted in higher air fraction from 5 min whipping. We suggest that in our case increased foamability at lower temperatures $\left(<-5^{\circ} \mathrm{C}\right)$ arises from an increase in $G^{\prime}$ of oil gels before aeration. In foaming of RPO upon warming (open points), the variation of over-run with aeration temperature is similar but with higher values. The difference may be attributed to different crystal sizes and polymorphs induced by the two tempering procedures, as reported in previous studies ${ }^{35,55}$ since melting-mediated tempering tends to yield smaller $\beta$-fat crystals resulting in better foamability. The effect of whipping temperature on the over-run of whipped EVOO also displays a maximum as shown in Figure 6(b). The highest over-run on cooling $(\sim 110 \%)$ is achieved near $T_{\mathrm{g}}$, i.e. $-12{ }^{\circ} \mathrm{C}$ and $\Delta T=-3{ }^{\circ} \mathrm{C}$. During heating, foaming can be achieved over a wider range of temperature, and the maximum is shifted to a higher temperature. The inset in Figure 6(b) is the appearance of EVOO foam fabricated at $-12{ }^{\circ} \mathrm{C}$ during cooling.

A noticeable difference exists between RPO and EVOO regarding foaming capacity. The maximum over-run of whipped EVOO is $\sim 110 \%$, much higher than that of $\sim 38 \%$ for whipped RPO. The maximum volume fraction of air for EVOO foams is $\sim 0.55$ which is similar to that in previous work $^{27,33,36}$ and close to the random close packing fraction of monodisperse hard spheres of 0.64 . One possible reason for the difference is that the size and morphology of RPO and EVOO crystals differ (Figure 3). ${ }^{38}$ In addition, RPO is refined with minor polar components whereas EVOO is unrefined with a variety of polar components including free fatty acids, monoglycerides and polyphenols. ${ }^{61}$ We suggest that the driving force for the adsorption of triglyceride crystals to the air-EVOO interface is larger than that for RPO as the minor components of EVOO probably increase its polarity. ${ }^{35,62}$ Moreover, in oil foams stabilised by a mixture of fat and molecular foaming agent, interfacial templating crystallisation occurs, leading to smaller bubble size and improved foam stability. ${ }^{31}$ To test this hypothesis, raw EVOO was passed twice through a basic alumina column to remove polar impurities prior to whipping. As expected, whipped columned EVOO exhibits a 
significantly lower over-run (max. $\sim 50 \%$, see Figure S6) than that of raw EVOO (max. $\sim 110 \%, p<0.05$ ), supporting our suggestion.

Optical microscopy and cryo-SEM were used to investigate the microstructure of RPO and EVOO foams. As shown in Figure 7(a), RPO foam bubbles are polydisperse and spherical, and their surfaces are slightly rough and textured due to the adsorption of crystals, in line with the findings of Saremnejad et al. ${ }^{30}$ In the case of EVOO foams (Figure 7(b)), some bubbles are non-spherical however, implying jamming of trapped crystals at their surfaces hindering shape relaxation to spherical. ${ }^{33,35}$ In addition, a birefringent layer coating the bubbles can be identified by polarised microscopy. Cryo-SEM images of fresh foams at $-10{ }^{\circ} \mathrm{C}$ during cooling are given in Figure $7(\mathrm{c})$. They reveal that the surfaces of RPO foam bubbles are less textured than those of EVOO. It further suggests that the coverage of bubbles by crystals is higher for EVOO than RPO (see Figure 10 later). ${ }^{63}$ The average bubble diameter of aerated RPO decreases with decreasing whipping temperature, from $130 \mu \mathrm{m}$ at 3 ${ }^{\circ} \mathrm{C}$ to $80 \mu \mathrm{m}$ at $-7.5{ }^{\circ} \mathrm{C}$ (data not shown). By contrast, that for EVOO foams is $25 \mu \mathrm{m}$ and independent of aeration temperature, similar to that in Brun et al. ${ }^{27}(10-50 \mu \mathrm{m})$, Mishima et $a l .{ }^{35}(20-35 \mu \mathrm{m})$, Binks and Marinopoulos ${ }^{36}(20-30 \mu \mathrm{m})$ and Mishra et al. ${ }^{38}$ The arrangement of high-melting triglyceride crystals near the air-oil surface and in the continuous phase has been determined through elegant micro-XRD experiments. ${ }^{35}$ The faces of triglyceride crystals at bubble surfaces are oriented parallel to the air-oil surface, whilst those within the continuous oil phase are randomly arranged. We suggest from this that an energy-favourable configuration is such that the methyl groups of trigylceride crystal faces interact with both the air and oil phases, whereas their edges mainly composed of methylene and glycerol groups interact with other edges of adjacent adsorbed triglyceride crystals (via van der Waals forces and hydrogen bonds).

In order to evaluate foam stability, freshly whipped oils were stored at the respective whipping temperature, and their appearance over one month was recorded photographically and using optical microscopy. The appearance of RPO foams immediately and seven days after preparation is shown in Figure S7(a). For foams prepared and stored above $T_{\mathrm{g}}\left(-5^{\circ} \mathrm{C}\right)$, oil drains and accumulates at the vessel bottom; actually this process ceases within the first $24 \mathrm{~h}$ due to the strengthening of the fat crystal network with sintering. ${ }^{37,49}$ It is worth noting that the drained oil is slightly turbid, implying that not all the crystals are trapped within the foam. By contrast, no oil drains for oil foams prepared and stored at temperatures $\leq T_{\mathrm{g}}$. The relationship between the volume of oil drained after 7 days storage relative to the initial 
volume of foam and $G^{\prime}$ of unaerated samples is shown in Figure S7(b). Drainage decreases gradually with increasing $G$, from $8 \%$ at $3{ }^{\circ} \mathrm{C}$ to zero at $\leq-5{ }^{\circ} \mathrm{C}$ when $G$ reaches $10^{3} \mathrm{~Pa} .{ }^{27}$ Apart from liquid drainage, foam stability was also evaluated in terms of coarsening/coalescence. Optical micrographs of RPO foams initially and 7 days after preparation are shown in Figure S8. No coarsening/coalescence occurs at temperatures $\leq-5$ ${ }^{\circ} \mathrm{C}$ since the bubble size distribution remains similar to that initially $(p>0.05)$, whilst for $>-5$ ${ }^{\circ} \mathrm{C}$ the average bubble size increases gradually with storage time $(p<0.05)$. The evolution in the appearance of EVOO foams up to one month storage can be seen in Figure S9 (photos) and Figure S10 (micrographs). Although foams prepared at $-6{ }^{\circ} \mathrm{C}$ during cooling or at $0{ }^{\circ} \mathrm{C}$ during warming exhibit no drained oil, they suffer coarsening/coalescence as evidenced by a noticeable change in bubble size distribution during storage $(p<0.05)$. At temperatures $\leq-10$ ${ }^{\circ} \mathrm{C}$ oil foams are ultra-stable against drainage, coarsening and coalescence up to at least one month.

\subsection{Thermo-responsiveness of oil foams}

Stimuli-responsive foams are of particular interest in industry in the areas of controlled encapsulation, delivery and release. ${ }^{64}$ The stimuli can include $\mathrm{pH}$, temperature, magnetic field, ultrasound etc. In our systems, ultra-stable vegetable oil foams are stabilised by triglyceride crystals whose melting profile spans a wide range of temperature. Consequently, it is reasonable to predict that stable oil foams can be rendered unstable on heating, arising from the melting of crystals at both the interface and in bulk. To verify this, we chose to investigate the effect of heating on an otherwise stable RPO foam prepared at $-7.5{ }^{\circ} \mathrm{C}$ from cooling and an EVOO foam prepared at $-6{ }^{\circ} \mathrm{C}$ from warming. The foams were warmed progressively to specific temperatures at $1{ }^{\circ} \mathrm{C} \mathrm{min}^{-1}$. The effect of temperature on the appearance of the foams is shown in Figure 8. For RPO foam, there is no observable change in appearance between $-7.5{ }^{\circ} \mathrm{C}$ and $-5{ }^{\circ} \mathrm{C}$. The first sign of drainage occurs at $-3{ }^{\circ} \mathrm{C}$ at which a minor endothermic peak is observed from the melting profile of neat RPO. Upon warming further, the extent of oil leakage increases slightly. By $11^{\circ} \mathrm{C}$, i.e. at the major endothermic peak from the melting profile of neat RPO, the foam network collapses abruptly leaving a small volume of residual foam. The temperature for complete foam collapse is $15^{\circ} \mathrm{C}$ where all crystals melt. During heating, an increase in bubble size is evidenced by $-3{ }^{\circ} \mathrm{C}$ due to either or both of coarsening and coalescence. This is followed by a gradual increase in bubble size with increasing temperature until no bubbles remain at $15{ }^{\circ} \mathrm{C}$ or above. In the case of EVOO foam, no drainage is observed between $-6{ }^{\circ} \mathrm{C}$ and $2{ }^{\circ} \mathrm{C}$. The appearance of oil is first 
witnessed on the wall interior at $4{ }^{\circ} \mathrm{C}$ and the temperature for complete foam collapse is 14 ${ }^{\circ} \mathrm{C}$. The corresponding microscope images reveal that at temperatures lower than $0{ }^{\circ} \mathrm{C}$, there is virtually no change in bubble size distribution and non-spherical bubbles exist. Upon heating to $4{ }^{\circ} \mathrm{C}$, crystals at both the interface and bulk begin to melt, leading to bubble relaxation into spherical and a significant increase in average bubble diameter from $\sim 25 \mu \mathrm{m}$ to $\sim 100 \mu \mathrm{m}$. No bubbles exist at or above $14{ }^{\circ} \mathrm{C}$. The variation of the volume of oil foam and that of drained oil with temperature is given in Figure 9. When comparing the melting behaviour of RPO and EVOO foams, we find that the temperature range during which foams suffer significant collapse is much broader for the latter than the former, whilst the temperatures for complete foam collapse are comparable. Due to the reversible nature of triglyceride crystal melting and crystallisation, we chose to cool the molten RPO formed at $50{ }^{\circ} \mathrm{C}$ to $-7.5{ }^{\circ} \mathrm{C}$ and re-whipped it with the same protocol. The resulting over-run was comparable to that found initially, demonstrating the reversibility of the process.

\subsection{Determination of fatty acid composition in oil crystals}

RPO and EVOO are mainly composed of long-chain, unsaturated fatty acids (UFAs) in their triglyceride molecules. By selective crystallisation of high-melting triglyceride fractions within low-melting continuous oil, we have shown that such oil dispersions can be aerated producing stable oil foams. Foaming could be achieved over a broad range of temperature in which air bubbles are protected by adsorption of high-melting triglyceride crystals. However, we do not know the chemical composition of these crystals. In light of the above, we tried to separate the high-melting solid fractions from the liquid ones under vacuum filtration at $7.5 \pm 1{ }^{\circ} \mathrm{C}$ where the oils were partially crystallised. The recovery of RPO crystals was unsuccessful however since their crystal size was smaller than the pore size of the filter paper used, unlike the case for EVOO crystals. Thus, carefully collected EVOO crystals were subsequently melted and then converted into fatty acid methyl esters (FAMEs) by trans-esterification at room temperature. GC/MS analysis determined their fatty acid composition. Meanwhile, liquid EVOO was trans-esterified and analysed for comparison. The overlaid GC-MS chromatograms of liquid EVOO and high-melting EVOO crystals are given in Figure S11. They are similar with respect to both intensity and retention time. By comparing their MS spectra with library data (NIST), they are found to possess similar fatty acid types. The fatty acid proportions of both samples are listed in Table S2. Original EVOO contains $\mathbf{1 7 . 0 \%}$ SFAs and $80.8 \%$ UFAs. For high-melting EVOO crystals, the SFAs increase 
to $23.0 \%$ and that of UFAs decrease to $75.1 \%(p<0.05)$. Thus the ratio of SFA/UFA increases from 21.0 in liquid EVOO to 30.6 in EVOO crystals, an increase of $46 \%$. This means that a higher proportion of SFAs is present in EVOO crystals compared with original EVOO oil, ${ }^{45,65}$ in support of our initial hypothesis that high-melting saturated fractions crystallise in a liquid of low-melting triglycerides.

\section{Conclusions}

Our initial hypothesis that a vegetable oil, capable of forming crystals by careful selection of temperature, can be aerated to produce an oil foam has been confirmed. We introduce a new protocol to prepare edible oil foams from vegetable oils containing mainly long-chain, UFAs in their triglyceride molecules in the absence of an added foaming agent. This is in contrast to earlier work in which amphiphilic molecules are added as foaming agent. $^{27-29,31,33,35}$ Careful temperature control induces the formation of high-melting triglyceride crystals within low-melting continuous liquid oil capable of forming an oleogel. Upon whipping, ultra-stable oil foams containing adsorbed crystals can be fabricated. The highest over-run is $\sim 40 \%$ at $\leq-16{ }^{\circ} \mathrm{C}$ for refined peanut oil and $\sim 110 \%$ at $-12{ }^{\circ} \mathrm{C}$ for extra virgin olive oil. We hypothesise that in RPO foams triglyceride crystals are loosely packed at the interface, whilst in EVOO foams a more coherent crystal layer forms at bubble surfaces (Figure 10). It is shown that triglyceride crystals contain a higher proportion of saturated fatty acids than the original oil. Such foams are temperature-responsive and can be rendered completely unstable upon heating to around the melting point of the crystals. We believe that our simple method is versatile and can be applied to a wide range of long-chain vegetable oils differing in fatty acid composition.

\section{Acknowledgements}

The authors thank the China Scholarship Council (CSC) for a PhD Scholarship to YL and Mr. T.S. Dunstan for assistance with cryo-SEM.

\section{Declaration of interest}

The authors confirm that there is no conflict of interest with regards to the work contained herein. 


\section{Author contributions}

BPB conceived the original idea, obtained funding and supervised the project. YL carried out all experiments. BPB and YL discussed and analysed the data and wrote the paper. 


\section{REFERENCES}

1. Campbell, G.M., \& Mougeot, E. (1999). Creation and characterisation of aerated food products. Trends Food Sci. Technol., 10, 283-296.

2. Garrett, P.R. (1993). Defoaming: theory and industrial applications. New York: Marcel Dekker, (Chapter 2).

3. Schramm, L. L. (1994). Foams: Fundamentals and Applications in the Petroleum Industry. Washington DC: American Chemical Society, (pp. 405-420).

4. Friberg, S.E. (2010). Foams from non-aqueous systems. Curr. Opin. Colloid Interface Sci., $15,359-364$.

5. Fameau, A-L., \& Saint-Jalmes, A. (2017). Non-aqueous foams: Current understanding on the formation and stability mechanisms. Adv. Colloid Interface Sci., 247, 454-464.

6. Binks, B. P. (2017). Colloidal Particles at a Range of Fluid-Fluid Interfaces. Langmuir, 33, 6947-6963.

7. Kovalchuk, N. M., Trybala, A., Starov, V., Matar, O., \& Ivanova, N. (2014). Fluoro- vs hydrocarbon surfactants: Why do they differ in wetting performance?. Adv. Colloid Interface Sci., 210, 65-71.

8. Bergeron, V., Hanssen, J. E., \& Shoghl, F. N. (1997). Thin-film forces in hydrocarbon foam films and their application to gas-blocking foams in enhanced oil recovery. Colloids Surf. A, 123-124, 609-622.

9. Ross, S., \& Nishioka, G. (1977). The relation of foam behaviour to phase separations in polymer solutions. Colloid Polymer Sci., 255, 560-565.

10. Friberg, S. E., Wohn, C. S., Greene, B., \& van Gilder, R. (1984). A non-aqueous foam with excellent stability. J. Colloid Interface Sci., 101, 593-595.

11. Binks, B. P., Davies, C. A., Fletcher, P. D. I., \& Sharp, E. L. (2010). Non-aqueous foams in lubricating oil systems. Colloids Surf. A, 360, 198-204.

12. Suja, V. C., Kar, A., Cates, W., Remmert, S. M., Savage, P. D., \& Fuller, G. G. (2018). Evaporation-induced foam stabilization in lubricating oils. PNAS, 115, 7919-7924.

13. Shrestha, L. K., Aramaki, K., Kato, H., Takase, Y., \& Kunieda, H. (2006). Foaming properties of monoglycerol fatty acid esters in nonpolar oil systems. Langmuir, 22, $8337-8345$.

14. Kunieda, H., Shrestha, L. K., Acharya, D. P., Kato, H., Takase Y., \& Gutierrez, J. M. (2007). Super-stable nonaqueous foams in diglycerol fatty acid esters-nonpolar oil systems. $J$. Disp. Sci. Technol., 28, 133-142. 
15. Shrestha, L. K., Shrestha, R. G., Sharma, S. C., \& Aramaki, K. (2008). Stabilization of nonaqueous foam with lamellar liquid crystal particles in diglycerol monolaurate/olive oil system. J. Colloid Interface Sci., 328, 172-179.

16. Shrestha, R. G., Shrestha, L. K., Solans, C., Gonzalez, G., \& Aramaki, K. (2010). Nonaqueous foam with outstanding stability in diglycerol monomyristate/olive oil system. Colloids Surf. A, 353, 157-165.

17. Fox, H. W., \& Zisman, W. A. (1950). The spreading of liquids on low energy surfaces. I. polytetrafluoroethylene. J. Colloid Interface Sci., 5, 514-531.

18. Fox, H. W., \& Zisman, W. A. (1952). The spreading of liquids on low-energy surfaces. II. Modified tetrafluoroethylene polymers. J. Colloid Interface Sci., 7, 109-121.

19. Murakami, R., \& Bismarck, A. (2010). Particle-Stabilized Materials: Dry Oils and (Polymerized) Non-Aqueous Foams. Adv. Funct. Mater., 20, 732-737.

20. Murakami, R., Kobayashi, S., Okazaki, M., Bismarck, A., \& Yamamoto, M. (2018). Effects of Contact Angle and Flocculation of Particles of Oligomer of Tetrafluoroethylene on Oil Foaming. Frontiers in Chem., 6, 435.

21. Binks, B. P., \& Rocher, A. (2010). Stabilisation of liquid-air surfaces by particles of low surface energy. Phys. Chem. Chem. Phys., 12, 9169-9171.

22. Binks, B. P., Rocher, A., \& Kirkland, M. (2011). Oil foams stabilized solely by particles. Soft Matter, 7, 1800-1808.

23. Binks, B. P., \& Tyowua, A. T. (2013). Influence of the degree of fluorination on the behaviour of silica particles at air-oil surfaces. Soft Matter, 9, 834-845.

24. Binks, B. P., Sekine, T., \& Tyowua, A. T. (2014). Dry oil powders and oil foams stabilised by fluorinated clay platelet particles. Soft Matter, 10, 578-589.

25. Binks, B. P., Johnston, S. K., Sekine, T., \& Tyowua, A. T. (2015). Particles at oil-air surfaces: Powdered oil, liquid oil marbles, and oil foam. ACS Appl. Mater. Interfaces, 7, 14328-14337.

26. Heymans, R., Tavernier, I., Dewettinck, K., \& van der Meeren, P. (2017). Crystal stabilization of edible oil foams. Trends Food Sci. Technol., 69, 13-24.

27. Brun, M., Delample, M., Harte, E., Lecomte, E., \& Leal-Calderon, F. (2015).

Stabilization of air bubbles in oil by surfactant crystals: a route to produce air-in-oil foams and air-in-oil-in-water emulsions. Food Res. Int., 67, 366-375.

28. Gunes, D. Z., Murith, M., Godefroid, J., Pelloux, C., Deyber, H., Schafer, O., \& Breton, O. (2017). Oleofoams: Properties of Crystal-Coated Bubbles from Whipped Oleogels-Evidence for Pickering Stabilization. Langmuir, 33, 1563-1575. 
29. Heymans, R., Tavernier, I., Danthine, S., Rimaux, T., van der Meeren, P., \& Dewettinck, K. (2018). Food-grade monoglyceride oil foams: the effect of tempering on foamability, foam stability and rheological properties. Food \& Function, 9, 3143-3154.

30. Saremnejad, F., Mohebbi, M., \& Koocheki, A. (2020). Practical application of nonaqueous foam in the preparation of a novel aerated reduced-fat sauce. Food and Bioproducts Processing, 119, 216-225.

31. Lei, M., Zhang, N., Lee, W.J., Tan, C.P., Lai, O.M., Wang, Y., \& Qiu, C. (2020). Non-aqueous foams formed by whipping diacylglycerol stabilized oleogel. Food Chem., 312, 126047.

32. Fameau, A-L., Lam, S., Arnould, A., Gaillard, C., Velev, O. D., \& Saint-Jalmes, A. (2015). Smart nonaqueous foams from lipid-based oleogel. Langmuir, 31, 13501-13510.

33. Binks, B. P., Garvey, E. J., \& Vieira, J. (2016). Whipped oil stabilised by surfactant crystals. Chem. Sci., 7, 2621-2632.

34. Truong, T., Prakash, S., \& Bhandari, B. (2019). Effects of crystallisation of native phytosterols and monoacylglycerols on foaming properties of whipped oleogels. Food Chem., 285, 86-93.

35. Mishima, S., Suzuki, A., Sato, K., \& Ueno, S. (2016). Formation and microstructures of whipped oils composed of vegetable oils and high-melting fat crystals. J. Am. Oil Chem. Soc., 93, 1453-1466.

36. Binks, B. P., \& Marinopoulos, I. (2017). Ultra-stable self-foaming oils. Food Res. Int., 95, 28-37.

37. Goibier, L., Pillement, C., Monteil, J., Faure, C., \& Leal-Calderon, F. (2019). Emulsification of non-aqueous foams stabilized by fat crystals: Towards novel air-in-oil-in-water food colloids. Food Chem., 293, 49-56.

38. Mishra, K., Dufour, D., \& Windhab, E. J. (2020). Yield Stress Dependent Foaming of Edible Crystal-Melt Suspensions. Cryst. Growth Des., 20, 1292-1301.

39. Mishra, K., Bergfreund, J., Bertsch, P., Fischer, P., \& Windhab, E. J. (2020). Crystallization-Induced Network Formation of Tri- and Monopalmitin at the Middle-Chain Triglyceride Oil/Air Interface, Langmuir, 36, 7566-7572.

40. Beltrán, G., del Rio, C., Sánchez, S., \& Martínez, L. (2004). Influence of Harvest Date and Crop Yield on the Fatty Acid Composition of Virgin Olive Oils from Cv. Picual. J. Agric. Food Chem., 52, 3434-3440. 
41. Kurata, S., Yamaguchi, K., \& Nagai, M. (2005). Rapid discrimination of fatty acid composition in fats and oils by electrospray ionization mass spectrometry. Anal. Sci., 21, 1457-1465.

42. Gribnau, M. C. M. (1992). Determination of solid/liquid ratios of fats and oils by low-resolution pulsed NMR. Trends Food Sci. Technol., 3, 186-190.

43. Macosko, C. W. (1994). Rheology: principles, measurements, and applications. New York: VCH.

44. Fitzgerald, A. M., Barnes, O. J., Smart, I., \& Wilson, D. I. (2001). Measurement of Particle Size Distribution of Tripalmitin Crystals in a Model Solution Using a Laser Diffraction Method. J. Am. Oil Chem. Soc., 78, 1013-1020.

45. Bootello, M. A., Garcés, R., Martínez-Force, E., \& Salas, J. J. (2011). Dry Fractionation and Crystallization Kinetics of High-Oleic High-Stearic Sunflower Oil. J. Am. Oil Chem. Soc., 88, 1511-1519.

46. European Union Commission. Regulation EEC 2568/91 on the characteristics of olive oil and olive pomace and their analytical methods (1991).

47. Valoppi, F., Calligaris, S., \& Marangoni, A. G. (2017). Structure and physical properties of oleogels containing peanut oil and saturated fatty alcohols. Eur. J. Lipid Sci. Technol., 119, 1600252 .

48. Marangoni, A. G. (2005). Fat Crystal Networks. Boca Raton: CRC Press, (Chapter 2).

49. Himawan, C., Starov, V. M., \& Stapley, A. G. F. (2006). Thermodynamic and kinetic aspects of fat crystallization. Adv. Colloid Interface Sci., 122, 3-33.

50. Chiavaro, E., Vittadini, E., Rodriguez-Estrada, M. T., Cerretani, L., Bonoli, M., Bendini, A., \& Lercker, G. (2007). Monovarietal Extra Virgin Olive Oils: Correlation Between Thermal Properties and Chemical Composition. J. Agric. Food Chem., 55, 10779-10786.

51. Barba, L., Arrighetti, G., \& Calligaris, S. (2013). Crystallization and melting properties of extra virgin olive oil studied by synchrotron XRD and DSC. Eur. J. Lipid Sci. Technol., 115, 322-329.

52. Andrikopoulos, N. K. (2002). Triglyceride species compositions of common edible vegetable oils and methods used for their identification and quantification. Food Rev. Int., 18, 71-102.

53. Che Man, Y. B., \& Tan, C. P. (2002). Comparative differential scanning calorimetric analysis of vegetable oils: II. Effects of cooling rate variation. Phytochemical Anal., 13, 142-151. 
54. Tan, C. P., \& Che Man, Y. B. (2002). Comparative differential scanning calorimetric analysis of vegetable oils: I. Effects of heating rate variation. Phytochemical Anal., 13, 129-141.

55. Higaki, K., Koyano, T., Hachiya, I., \& Sato, K. (2004). In situ optical observation of microstructure of $\beta$-fat gel made of binary mixtures of high-melting and low-melting fats. Food Res. Int., 37, 2-10.

56. Devi, A., \& Khatkar, B. S. (2018). Effects of fatty acids composition and microstructure properties of fats and oils on textural properties of dough and cookie quality. J. Food Sci. Technol., 55, 321-330.

57. Ojijo, N. K., Neeman, I., Eger, S., \& Shimoni, E. (2004). Effects of monoglyceride content, cooling rate and shear on the rheological properties of olive oil/monoglyceride gel networks. J. Sci. Food Agric., 84, 1585-1593.

58. Bals, A., \& Kulozik, U. (2003). The influence of the pore size, the foaming temperature and the viscosity of the continuous phase on the properties of foams produced by membrane foaming. J. Membr. Sci., 220, 5-11.

59. Krog, N., \& Larsson, K. (1992). Crystallization at interfaces in food emulsions - a general phenomenon. Eur. J. Lipid Sci. Technol., 94, 55-57.

60. Douaire, M., di Bari, V., Norton, J. E., Sullo, A., Lillford, P., \& Norton, I. T. (2014). Fat crystallisation at oil-water interfaces. Adv. Colloid Interface Sci., 203, 1-10.

61. Inglese, P., Famiani, F., Galvano, F., Servili, M., Esposto, S., \& Urbani, S. (2011). Factors affecting extra-virgin olive oil composition. Horticultural Rev., pp. 83-147.

62. de Vries, A., Gomez, Y. L., van der Linden, E., \& Scholten, E. (2017). The effect of oil type on network formation by protein aggregates into oleogels. RSC Adv., 7, 11803-11812.

63. Gautier, F., Destribats, M., Perrier-Cornet, R., Dechézelles, J., Giermanska, J., Héroguez, V., Ravaine, S., Leal-Calderon, F., \& Schmitt, V. (2007). Pickering emulsions with stimulable particles: from highly- to weakly-covered interfaces. Phys. Chem. Chem. Phys., 9, 6455-6462.

64. Fujii, S., \& Nakamura, Y. (2017). Stimuli-responsive bubbles and foams stabilized with solid particles. Langmuir, 33, 7365-7379.

65. Zaliha, O., Chong, C. L., Cheow, C. S., Norizzah, A. R., \& Kellens, M. J. (2004). Crystallization properties of palm oil by dry fractionation. Food Chem., 86, 245-250. 
Figure 1. Appearance of vessels containing (a) RPO and (b) EVOO upon cooling from $60{ }^{\circ} \mathrm{C}$ at $0.3{ }^{\circ} \mathrm{C} \mathrm{min}^{-1}$. Inset: tilted vessel. Scale bars $=1 \mathrm{~cm}$.

(a)

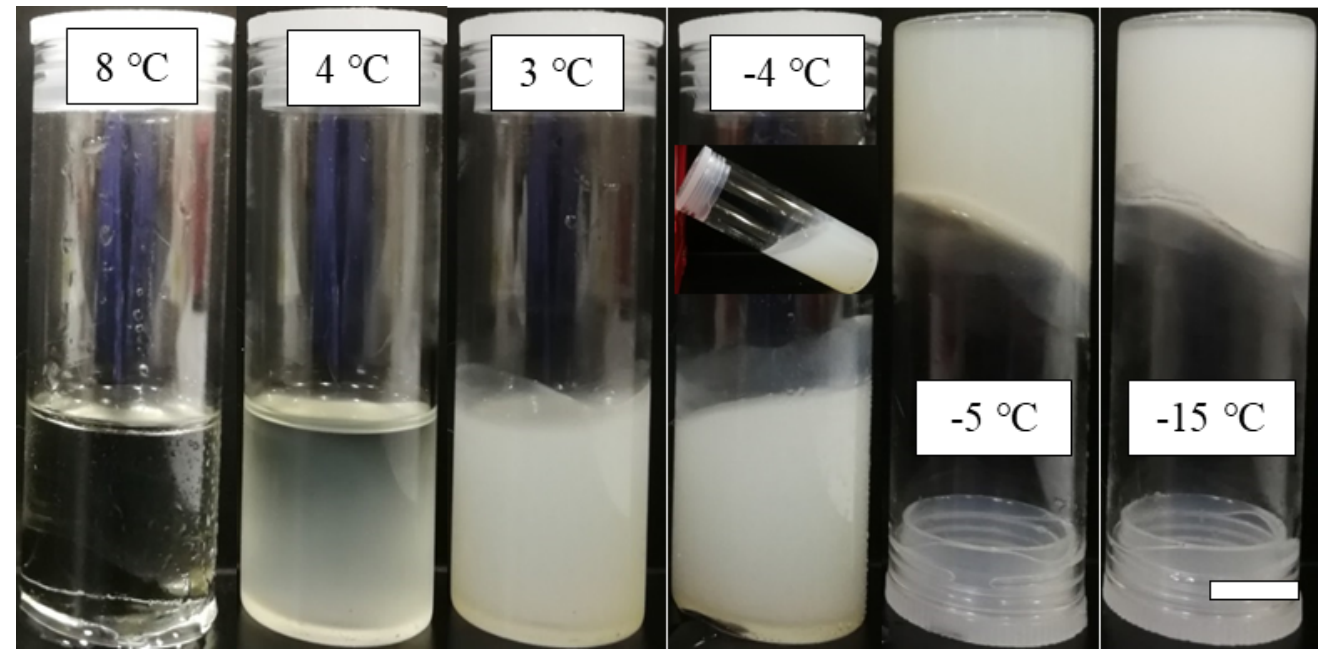

(b)

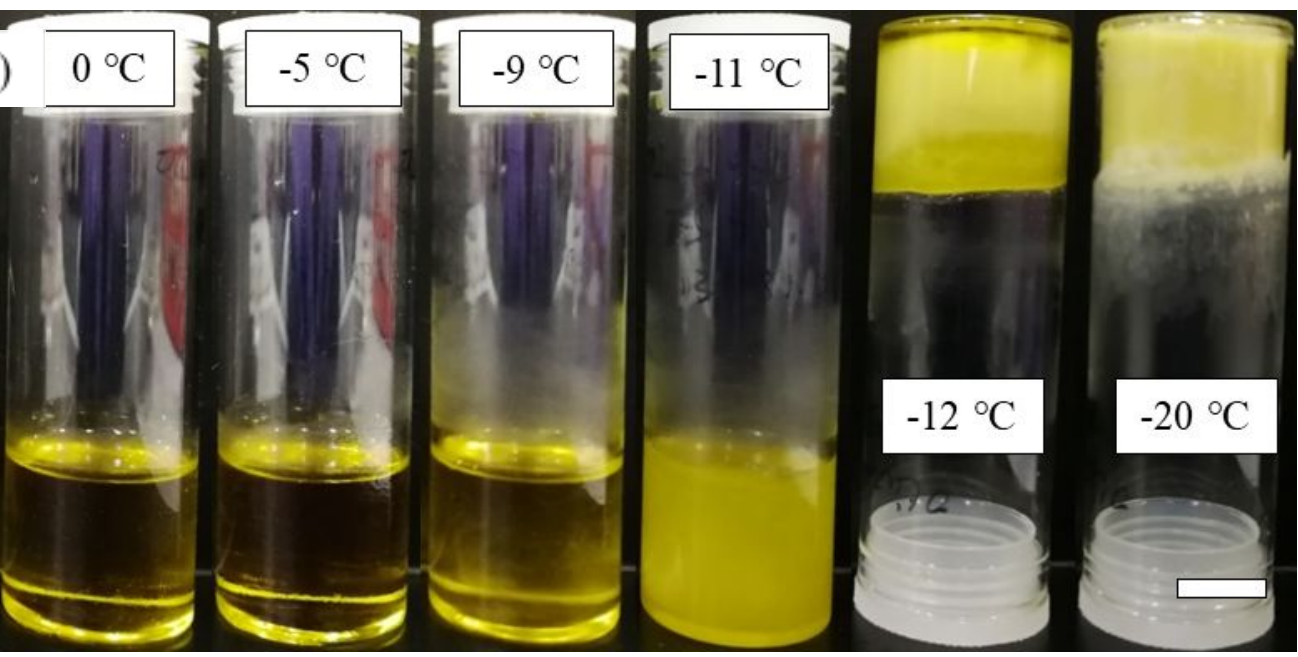


Figure 2. DSC curves for (a) RPO and (b) EVOO upon cooling (lower) and subsequent warming (upper). The cooling and warming rates were $0.5{ }^{\circ} \mathrm{C} \mathrm{min}^{-1}$. (c) Variation of SFC with temperature for RPO (open points) and groundnut oil (filled points). Tests were performed in triplicate.
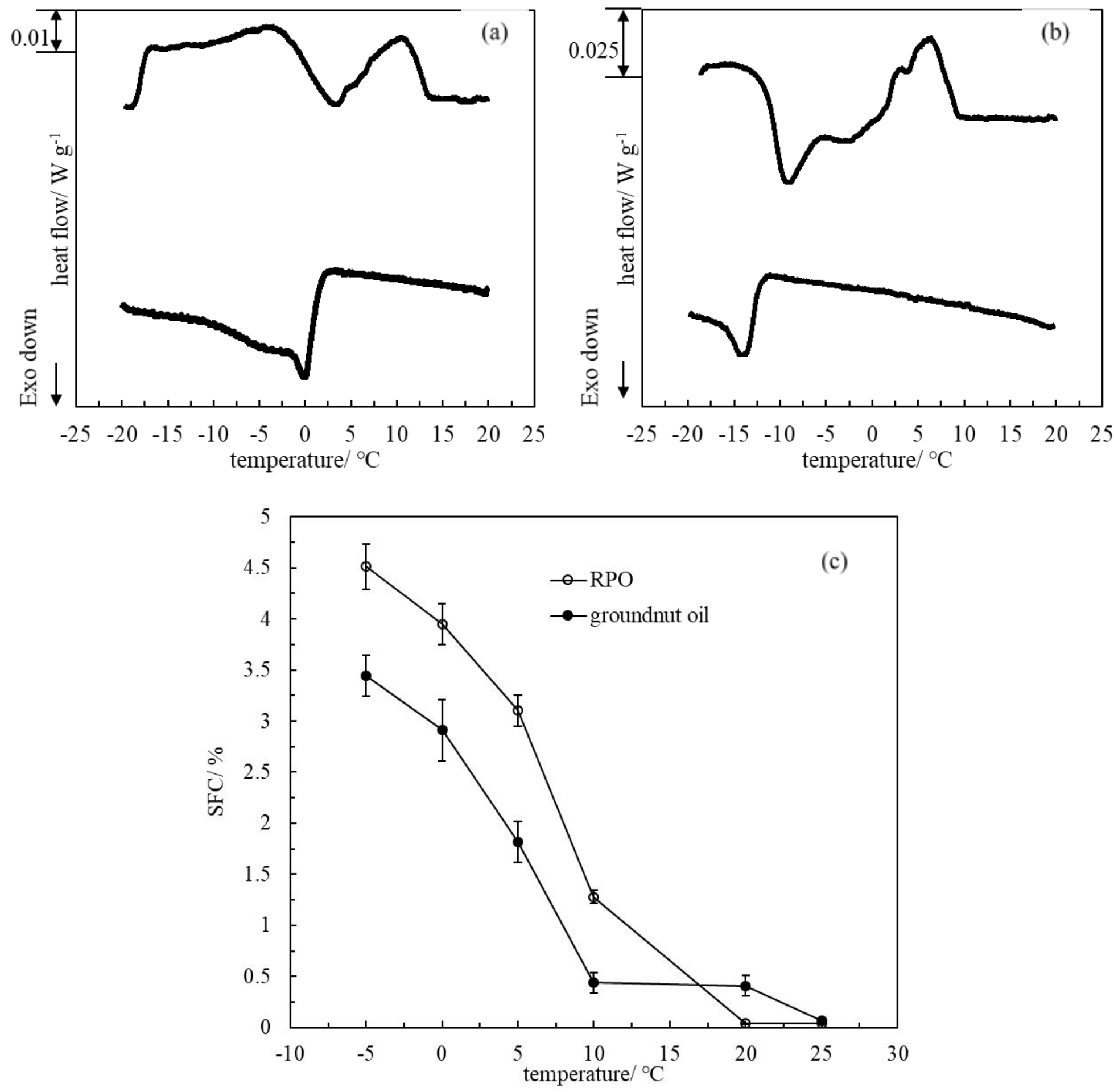
Figure 3. Polarised micrographs of (a) RPO and (b) EVOO at certain temperatures upon cooling from $60{ }^{\circ} \mathrm{C}$ at $0.3{ }^{\circ} \mathrm{C} \mathrm{min}^{-1}$. Scale bars $=50 \mu \mathrm{m}$.

(a)
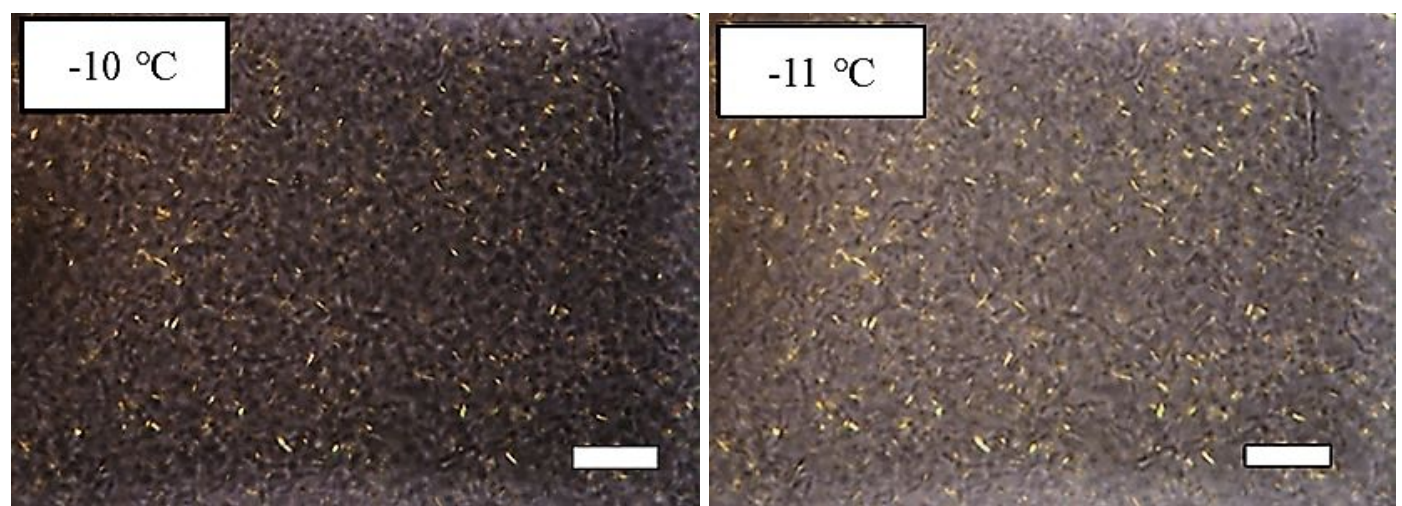

(b)
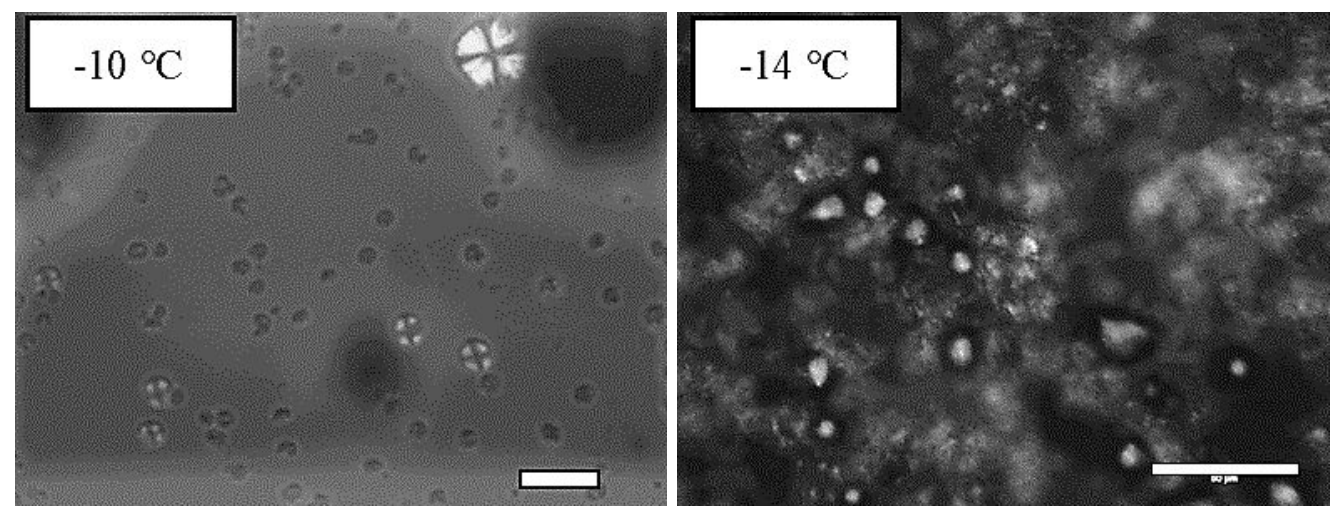
Figure 4. Variation of $G^{\prime}$ (triangles) and $G^{\prime \prime}$ (squares) upon cooling (open points) and subsequent warming (filled points) of (a) RPO and (b) EVOO. The cooling and warming rates were $1{ }^{\circ} \mathrm{C} \mathrm{min}^{-1}$. Lines are: $T_{\mathrm{cc}}$ - crossover temperature $\left(G^{\prime}=G^{\prime \prime}\right)$ of crystallisation during cooling; $T_{\mathrm{oc}}$ - onset temperature of crystallisation during cooling; $T_{\mathrm{cm}}$ - crossover temperature $\left(G^{\prime}=G^{\prime \prime}\right)$ of crystal melting during warming; $T_{\mathrm{em}}$ - end temperature of melting during warming. Tests were performed in triplicate.
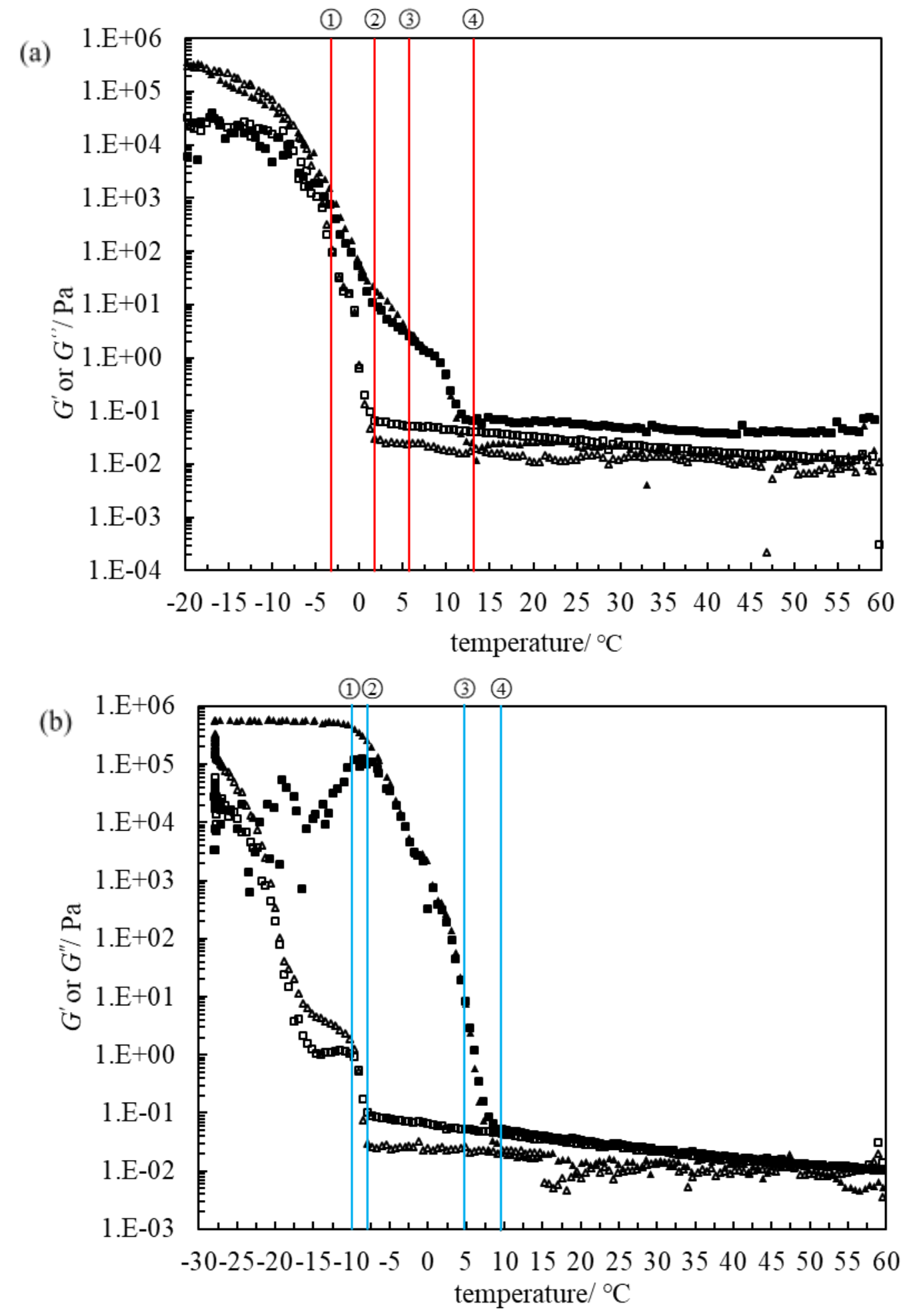
Figure 5. Appearance of oils as a function of whipping time at representative temperatures cooled from $60{ }^{\circ} \mathrm{C}$ at $0.3{ }^{\circ} \mathrm{C} \mathrm{min}^{-1}$. (a) RPO at $3{ }^{\circ} \mathrm{C}$ and (b) EVOO at $-10{ }^{\circ} \mathrm{C}$. The degree of supercooling $\Delta T=-1{ }^{\circ} \mathrm{C}$ for both systems. Inset: photos taken from above. Scale bars $=1 \mathrm{~cm}$. Over-run versus whipping time for (c) RPO and (d) EVOO at particular temperatures cooled from $60{ }^{\circ} \mathrm{C}$ at $0.3{ }^{\circ} \mathrm{C} \mathrm{min}^{-1}$. Tests were performed in at least triplicate.

(a)

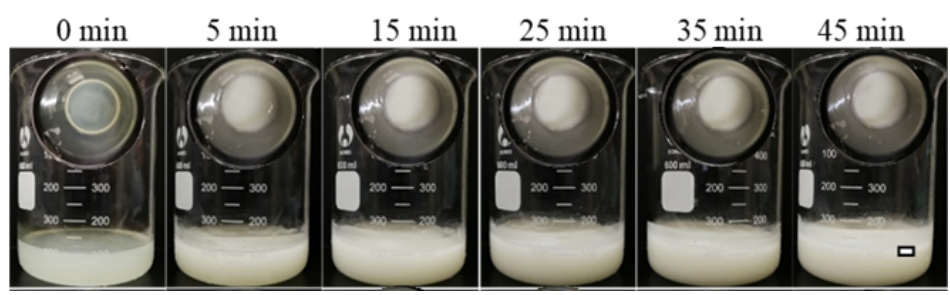

(b)

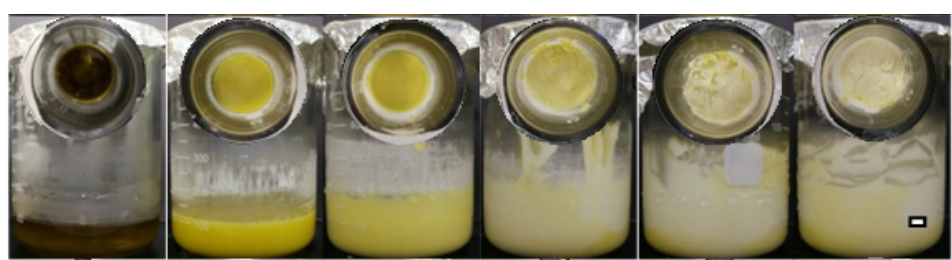




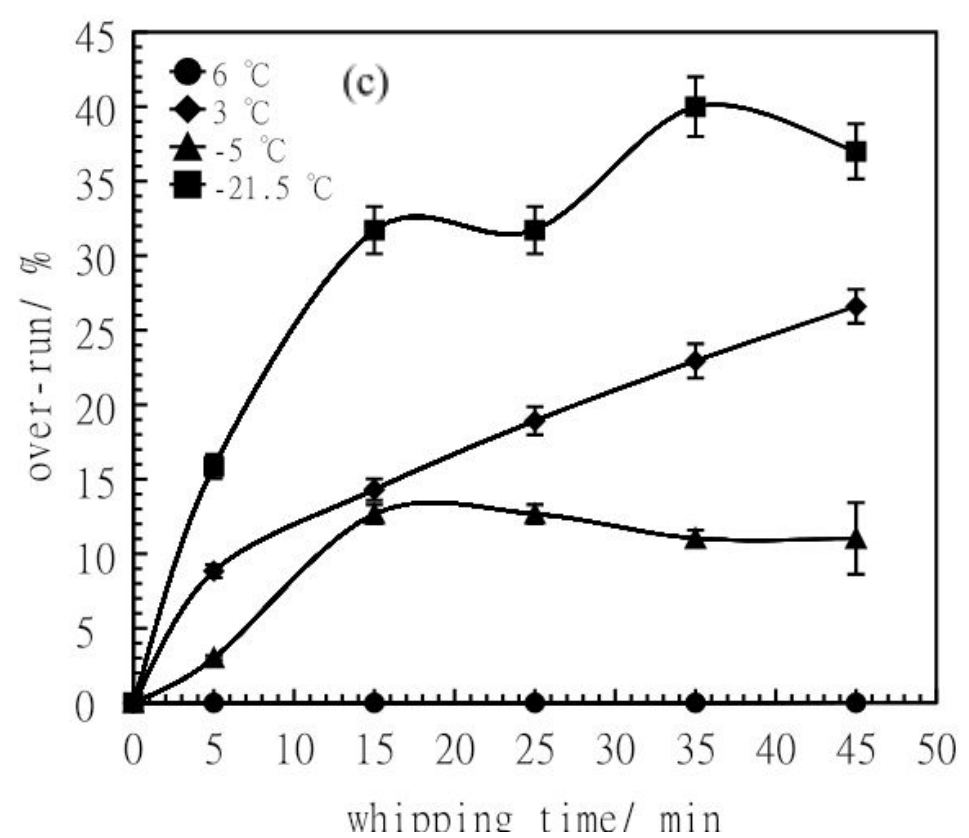

(d)

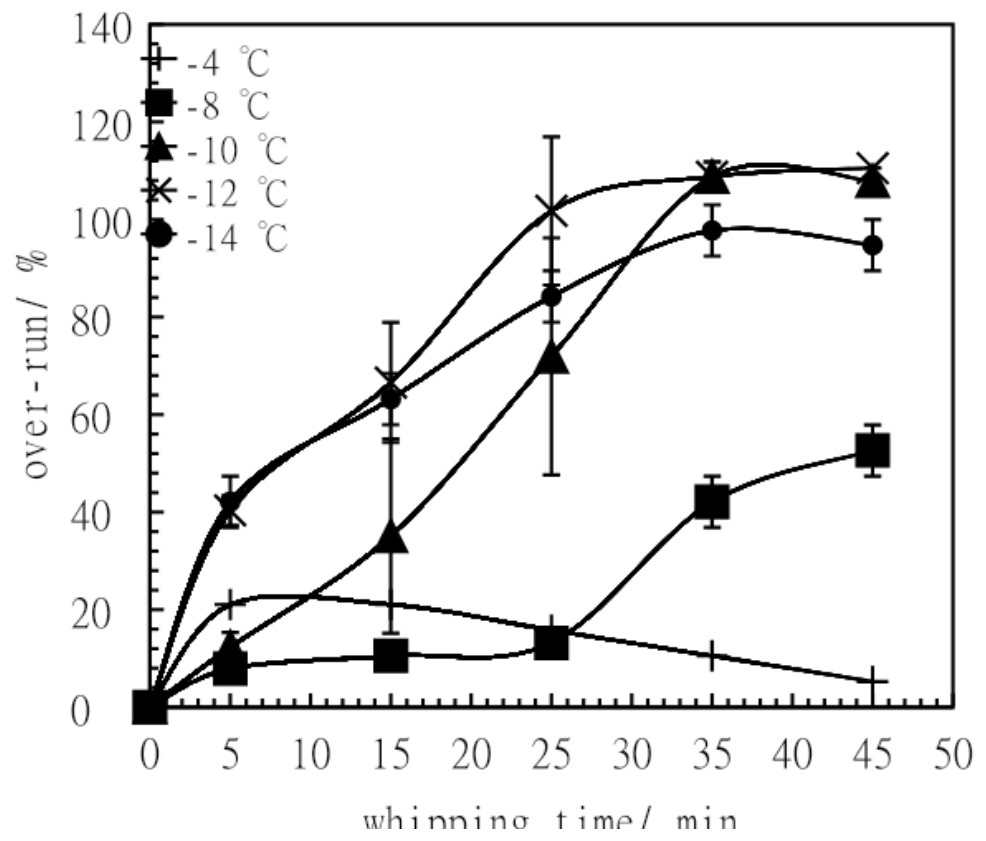


Figure 6. Over-run of (a) RPO foams and (b) EVOO foams after 45 min whipping as a function of whipping temperature upon cooling (filled points) and warming (open points). Inset: photos of RPO foams at $-15{ }^{\circ} \mathrm{C}$ and EVOO foams at $-12{ }^{\circ} \mathrm{C}$ cooled from $60{ }^{\circ} \mathrm{C}$ at $0.3{ }^{\circ} \mathrm{C}$ $\min ^{-1}$. Tests were performed in at least triplicate.

(a)

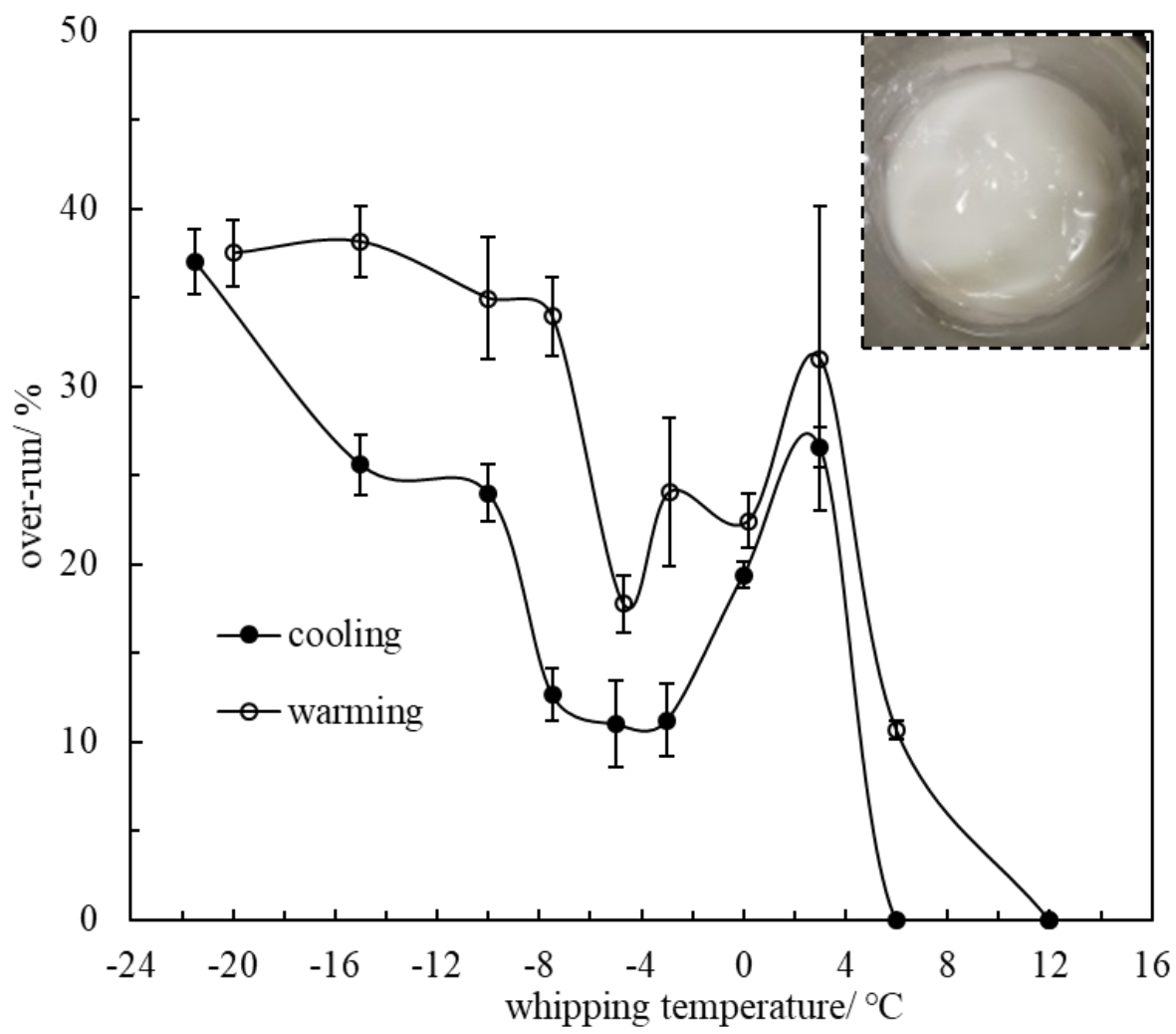

(b)

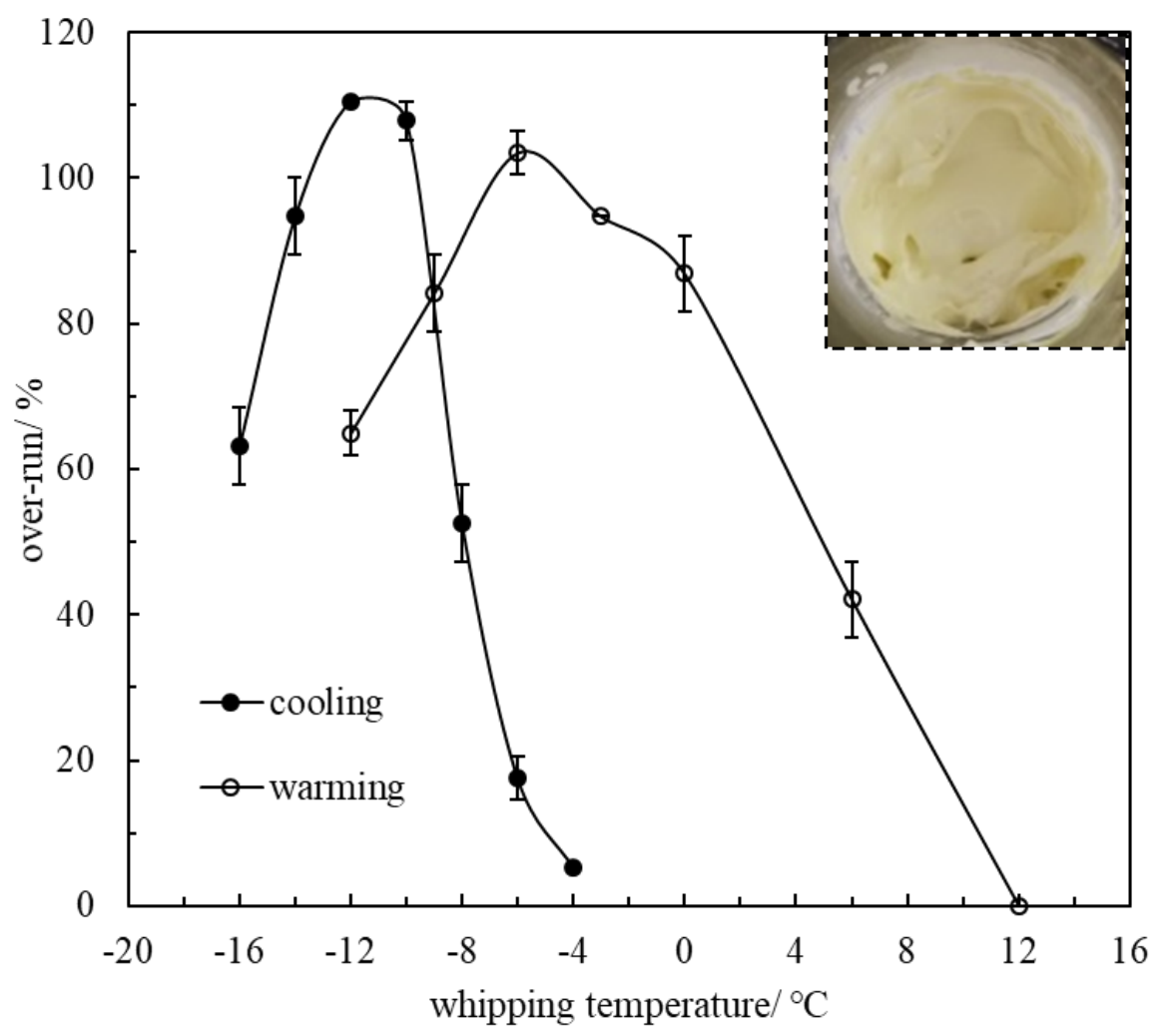


Figure 7. Optical micrographs of (a) RPO foams and (b) EVOO foams immediately after 45 min whipping at particular temperatures cooled from $60{ }^{\circ} \mathrm{C}$ at $0.3{ }^{\circ} \mathrm{C} \mathrm{min}{ }^{-1}$. Non-polarised micrographs for $0{ }^{\circ} \mathrm{C}$ and $-10{ }^{\circ} \mathrm{C}$, polarised micrographs for $-7.5{ }^{\circ} \mathrm{C}$ and $-6{ }^{\circ} \mathrm{C}$. Scale bars equal $50 \mu \mathrm{m}$ for RPO foams and $100 \mu \mathrm{m}$ for EVOO foams. (c) Cryo-SEM images of RPO foam (left) and EVOO foam (right) prepared at $-10{ }^{\circ} \mathrm{C}$ during cooling.

(a)
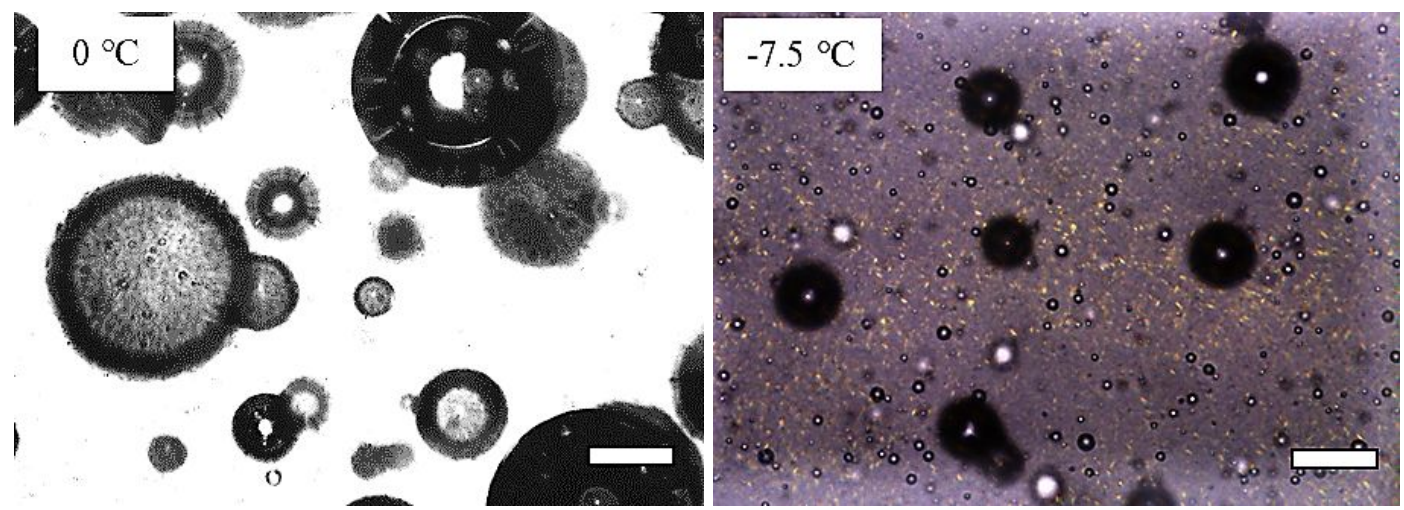

(b)
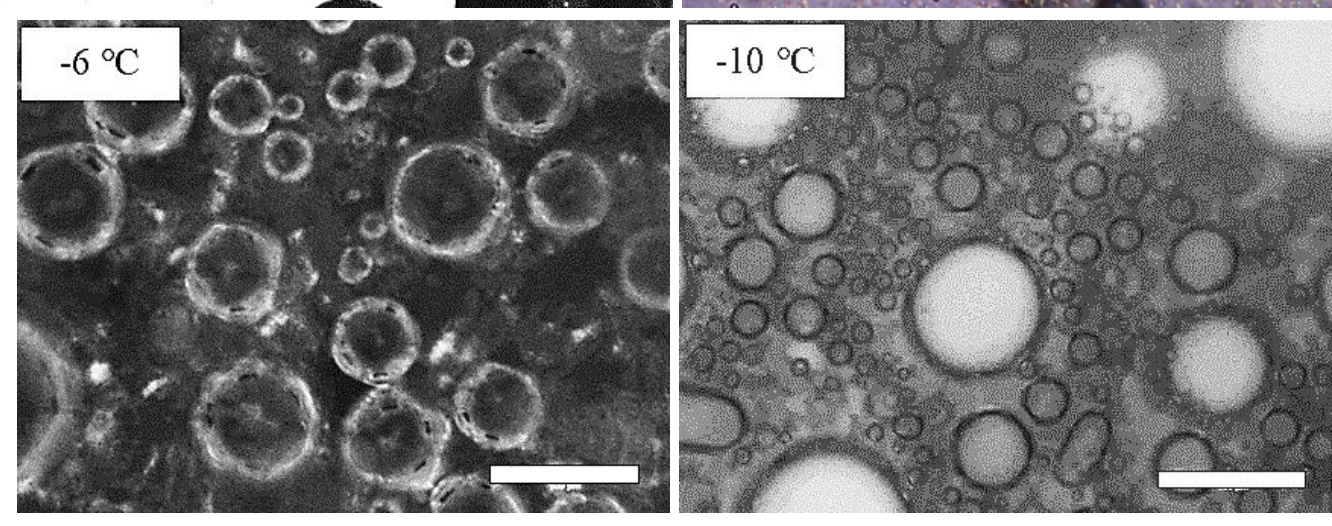

(c)
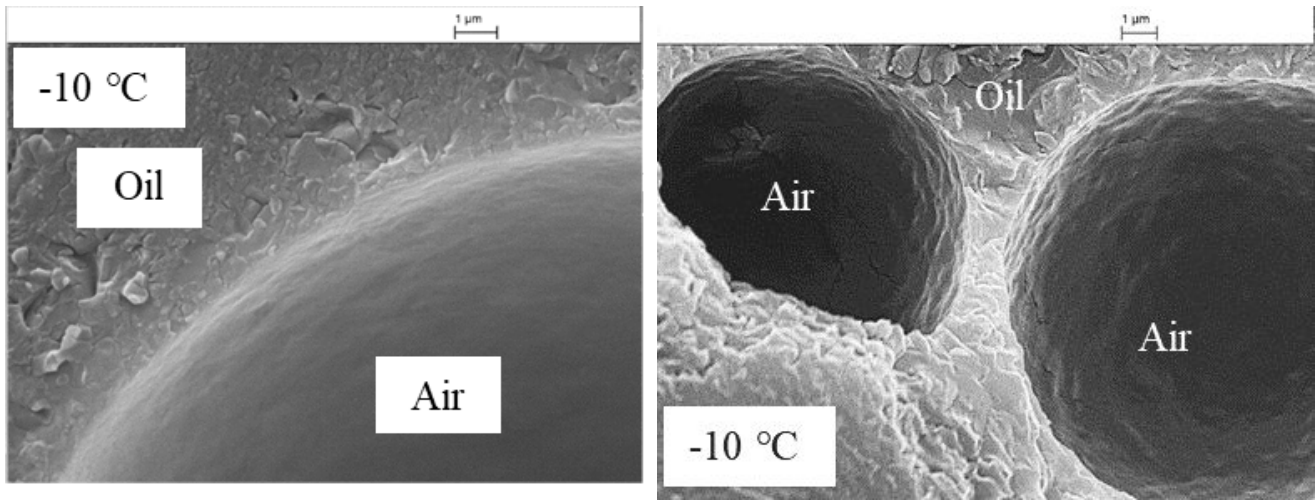
Figure 8. Effect of temperature on (a) appearance of RPO foams (upper) and EVOO foams (lower); heating rate was $1{ }^{\circ} \mathrm{C} \mathrm{min}^{-1}$, scale bars $=1 \mathrm{~cm}$ and (b) micrographs; scale bars $=500$ $\mu \mathrm{m}$ for RPO foams except at $50{ }^{\circ} \mathrm{C}$ which is $100 \mu \mathrm{m}$, scale bars $=100 \mu \mathrm{m}$ for EVOO foams. RPO foams were prepared at $-7.5{ }^{\circ} \mathrm{C}$ after cooling from $60{ }^{\circ} \mathrm{C}$ at $0.3{ }^{\circ} \mathrm{C} \mathrm{min}^{-1}$. EVOO foams were prepared at $-6{ }^{\circ} \mathrm{C}$ after warming from $-20{ }^{\circ} \mathrm{C}$ at $0.4{ }^{\circ} \mathrm{C} \mathrm{min}-1$.

(a) $\quad-7.5^{\circ} \mathrm{C} \quad-3{ }^{\circ} \mathrm{C} \quad 3{ }^{\circ} \mathrm{C} \quad 6{ }^{\circ} \mathrm{C} \quad 11^{\circ} \mathrm{C} \quad 50{ }^{\circ} \mathrm{C}$

(b)
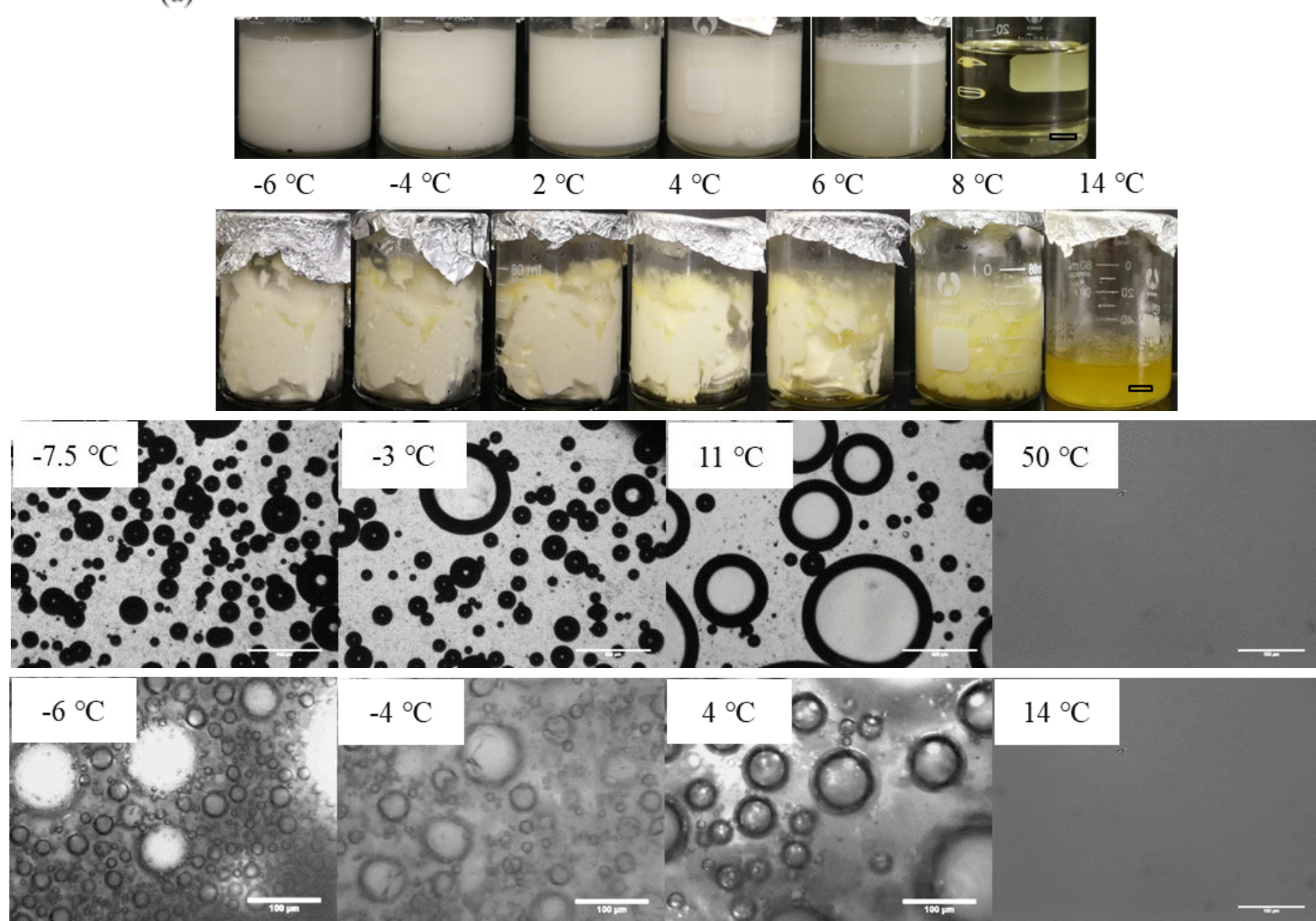
Figure 9. Variation of volume of foam (open points) and volume of drained oil (filled points) with temperature upon heating. Heating rate was $1{ }^{\circ} \mathrm{C} \mathrm{min}^{-1}$. RPO foams were prepared at $-7.5{ }^{\circ} \mathrm{C}$ after cooling from $60{ }^{\circ} \mathrm{C}$ at $0.3{ }^{\circ} \mathrm{C} \mathrm{min}^{-1}$. EVOO foams were prepared at $-6{ }^{\circ} \mathrm{C}$ after warming from $-20{ }^{\circ} \mathrm{C}$ at $0.4{ }^{\circ} \mathrm{C} \mathrm{min}^{-1}$. Tests were performed in triplicate.

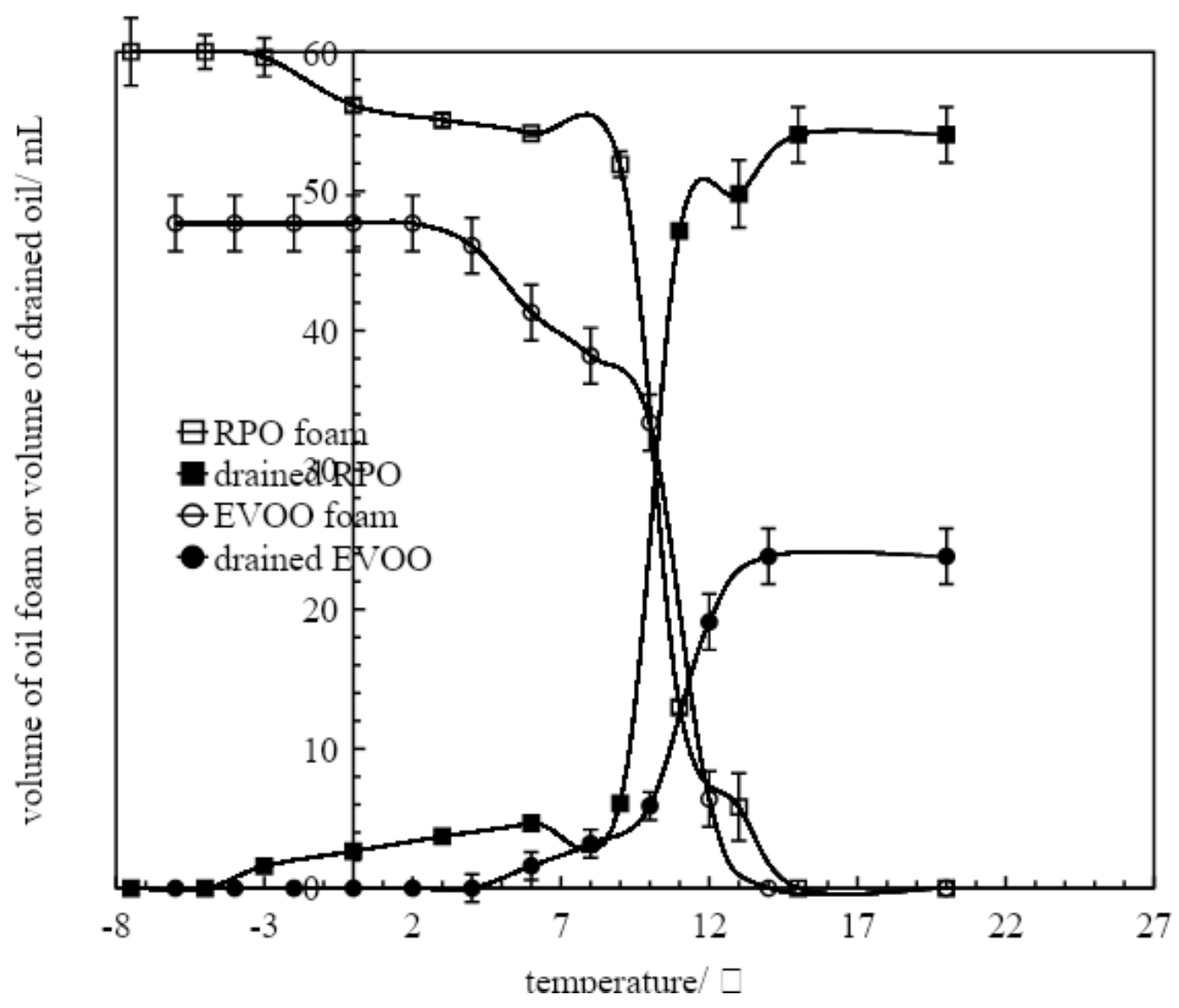


Figure 10. Schematic illustration of the stabilization of air-in-oil foams by long-chain triglyceride crystals. (a) RPO, (b) EVOO.
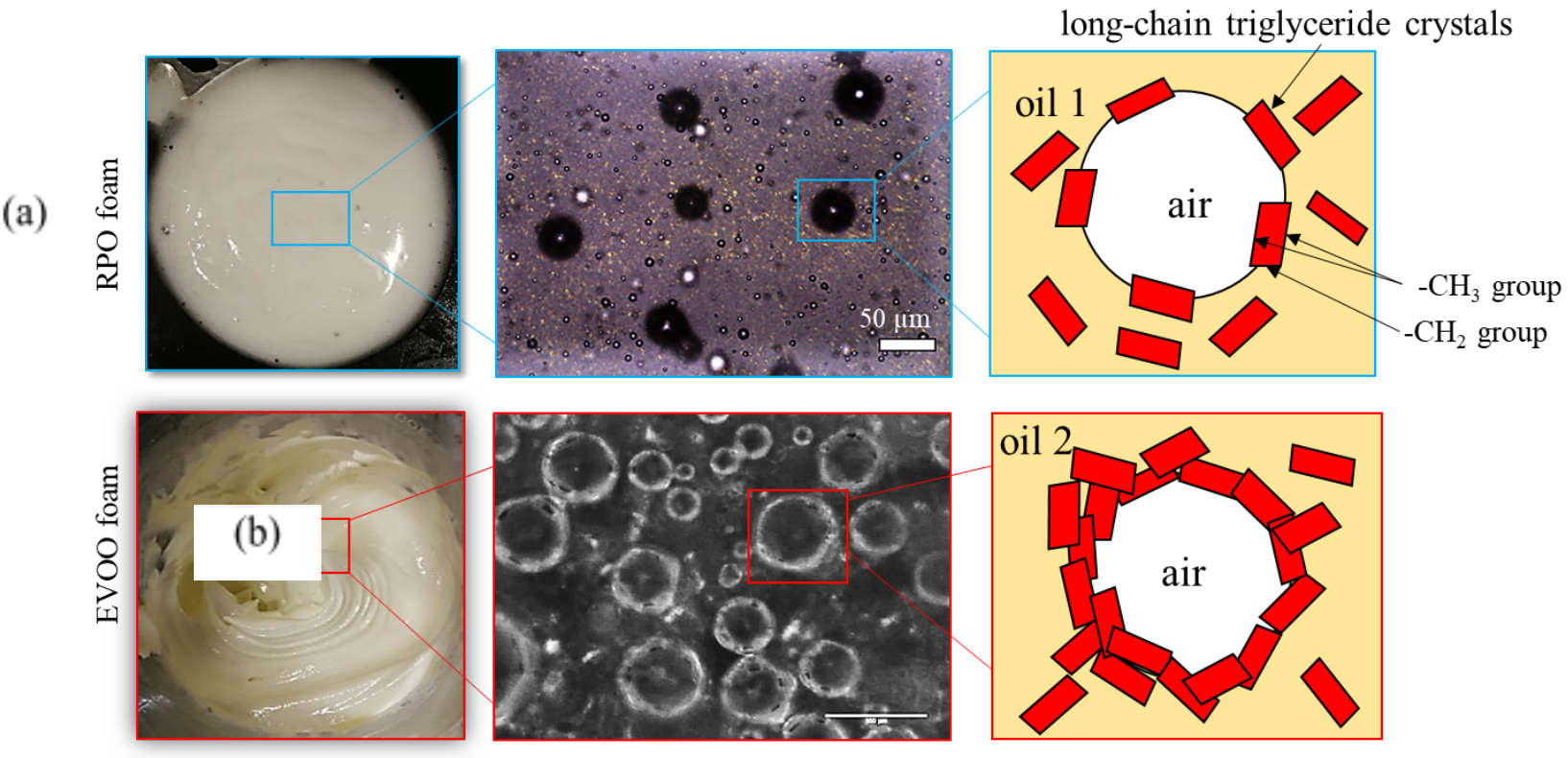